\title{
Transcriptome analysis of alternative splicing-coupled nonsense-mediated mRNA decay in human cells reveals broad regulatory potential
}

Courtney E. French ${ }^{1, \# a^{*}}$, Gang Wei ${ }^{2, \# b^{*}}$, James P. B. Lloyd ${ }^{2,3, \# c}$, Zhiqiang $\mathrm{Hu}^{2}$, Angela N. Brooks ${ }^{1, \# d}$, Steven E. Brenner ${ }^{1,2,3, \$}$

${ }^{1}$ Department of Molecular and Cell Biology, University of California, Berkeley, CA, 94720, USA

${ }^{2}$ Department of Plant and Microbial Biology, University of California, Berkeley, CA, 94720, USA

${ }^{3}$ Center for RNA Systems Biology, University of California, Berkeley, CA, 94720, USA

\#a Current address: Department of Paediatrics, University of Cambridge, Cambridge, CB2 1TN, UK

\#b Current address: State Key Laboratory of Genetics Engineering \& MOE Key Laboratory of Contemporary Anthropology, School of Life Sciences, Fudan University, Shanghai, 200433, China

\#c Current address: ARC Centre of Excellence in Plant Energy Biology, University of Western Australia, Perth, Australia

${ }^{\# d}$ Current address: Department of Biomolecular Engineering, University of California, Santa Cruz, CA, USA

${ }^{*}$ These authors contributed equally to this work

\$Correspondence: brenner@compbio.berkeley.edu 


\section{Abstract:}

To explore the regulatory potential of nonsense-mediated mRNA decay (NMD) coupled with alternative splicing, we globally surveyed the transcripts targeted by this pathway via RNASeq analysis of HeLa cells in which NMD had been inhibited. We identified putative NMDtargeted transcripts as those with a termination codon more than 50 nucleotides upstream of an exon-exon junction (premature termination as defined by the '50nt rule') and that significantly increased in abundance upon NMD inhibition. We additionally controlled for potential transcriptional up-regulation by requiring the putative NMD targets to increase in abundance substantially more than the isoforms from the same gene that do not contain a premature termination codon. This resulted in a conservative set of 2,793 transcripts derived from 2,116 genes as physiological NMD targets $(9.2 \%$ of expressed transcripts and $>20 \%$ of alternatively spliced genes). Our analysis identified previously inferred unproductive isoforms and numerous heretofore-uncharacterized ones. NMD-targeted transcripts were derived from genes involved in many functional categories, and are particularly enriched for RNA splicing genes as well as for those harboring ultraconserved elements. By investigating the features of all transcripts impacted by NMD, we find that the 50nt rule is a strong predictor of NMD degradation while 3' UTR length on its own generally has only a small effect in this human cell line. Additionally, thousands more transcripts without a premature termination codon in the main coding sequence contain a uORF and display significantly increased abundance upon NMD inhibition indicating potentially widespread regulation through decay coupled with uORF translation. Our results support that alternative splicing coupled with NMD is a prevalent post-transcriptional mechanism in human cells with broad potential for biological regulation. 


\section{Introduction}

Nonsense-mediated mRNA decay (NMD) is a eukaryotic mRNA surveillance pathway with a role in gene regulation. In its surveillance capacity, NMD recognizes premature termination codons (PTCs) generated by nonsense mutations or errors in transcription and splicing and eliminates the aberrant transcripts in order to protect the cell from the production of potentially harmful truncated proteins (Frischmeyer-Guerrerio et al. 2011; He et al. 1993; Isken and Maquat 2007; Kervestin and Jacobson 2012; Lykke-Andersen and Bennett 2014; Pulak and Anderson 1993). Many other transcripts targeted to NMD are generated by highly regulated and evolutionarily conserved splicing events (Lareau, Inada, et al. 2007; Lareau and Brenner 2015; Mudge et al. 2011; Ni et al. 2007; Yan et al. 2015). This coupling of alternative splicing and NMD (AS-NMD) can be used to post-transcriptionally regulate the level of mRNA (also termed Regulated Unproductive Splicing and Translation, or RUST) (He and Jacobson 2015; Kurosaki, Popp, and Maquat 2019; Lewis, Green, and Brenner 2003; Lykke-Andersen and Jensen 2015; Rehwinkel et al. 2005; Schweingruber et al. 2013).

A number of splicing factors are known to auto-regulate their own expression or regulate the expression of other splicing factors through alternative splicing coupled with NMD (Änkö et al. 2012; Dredge and Jensen 2011; Jangi et al. 2014; Jumaa and Nielsen 1997; McGlincy et al. 2010; Pervouchine et al. 2019; Rossbach et al. 2009; Saltzman et al. 2008; Saltzman, Pan, and Blencowe 2011; Spellman, Llorian, and Smith 2007; Sun et al. 2010; Sureau et al. 2001). Many of these NMD-coupled splicing events (including all those in the SR gene family) are associated with a highly conserved or ultraconserved element (Bejerano et al. 2004) of the human genome, indicating a potential role for these elements in the regulation of alternative splicing coupled with NMD (Lareau, Brooks, et al. 2007; Ni et al. 2007). Genes with other functions have also been 
reported to be regulated via alternative splicing coupled to NMD such as the spermidine/spermine N1-acetyltransferase (SSAT) gene (Hyvönen et al. 2006, 2012) and ribosomal proteins (Cuccurese et al. 2005; Plocik and Guthrie 2012; Russo et al. 2011; Zheng et al. 2012). In addition, NMD, not always coupled to alternative splicing, has been shown to be important for multiple differentiation programs including neuronal, granulocyte, and erythropoiesis differentiation (Bruno et al. 2011; Karam and Wilkinson 2012; Lasalde et al. 2014; Pimentel et al. 2014; Weischenfeldt et al. 2008; Wong et al. 2013), essential for viability via regulation of GADD45, a stress and DNA-damage response factor (Nelson et al. 2016), and playing a role in cancer (Fernandes et al. 2019).

Numerous studies have been performed to investigate the components modulating the NMD process, resulting in identification of many NMD factors (Bhattacharya et al. 2000; Deniaud et al. 2015; Gehring et al. 2005; Huntzinger et al. 2008; Ivanov et al. 2008; Kashima et al. 2006; Lloyd et al. 2018; Lykke-Andersen and Bennett 2014; Lykke-Andersen, Shu, and Steitz 2000; Melero et al. 2014; Yamashita et al. 2009). Among those proteins known to mediate the NMD pathway, UPF1 is a crucial and conserved component (Chakrabarti et al. 2011, 2014; Hwang et al. 2010; Lasalde et al. 2014; Nicholson et al. 2014; Okada-Katsuhata et al. 2012). However, other than UPF1, other NMD factors may be exclusive to different branches of the pathway (Metze et al. 2013) and the specific mechanisms of NMD and the defining features of NMD targets are still not completely understood and likely vary across different species (Kerényi et al. 2008; Lloyd 2018). In mammals, a prominent model posits that the presence of an exon-exon junction more than 50 nucleotides downstream of a stop codon induces NMD; this has been termed the "50 nucleotide rule" (Nagy and Maquat 1998; Zhang and Maquat 1997). The exon junction complex is a protein complex deposited near an exon-exon junction during the splicing 
process and acts as a cellular memory of an intron's location following splicing. When a ribosome terminates upstream of an exon-exon junction, NMD factors in the exon junction complex recruit UPF1, triggering decay (Chamieh et al. 2008). Long 3' UTRs have also been found to target transcripts to NMD (Amrani et al. 2004; Balagopal and Beemon 2017; Bao et al. 2016; Bühler et al. 2006; Chakrabarti et al. 2014; Fanourgakis et al. 2016; Ge et al. 2016; Hurt, Robertson, and Burge 2013; Kervestin et al. 2012; Kervestin and Jacobson 2012; Kishor, Fritz, and Hogg 2019; Kishor, Ge, and Hogg 2019; Kurosaki and Maquat 2013; Lareau and Brenner 2015; Nyikó et al. 2017; Singh, Rebbapragada, and Lykke-Andersen 2008; Toma et al. 2015; Wright et al. 2015; Yepiskoposyan et al. 2011). A long 3' UTR, or "faux" 3' UTR, has been proposed to trigger NMD in a couple of ways including structural characteristics, such as the long distance between PABP on the poly-A tail and the terminating ribosome, lead to aberrant termination (Behm-Ansmant et al. 2007; Eberle et al. 2008; Kervestin et al. 2012) or that UPF1 binds to 3' UTRs in a length-dependent manner and this primes the transcripts for NMD (Hogg and Goff 2010; Zünd et al. 2013).

The potential prevalence of NMD-targeted alternative isoforms was first established by searching EST databases for transcripts with a PTC (Lewis et al. 2003). These datasets, however, were generated from cells where NMD was active and the targets should be depleted. There have also been studies that successfully identified NMD-targeted transcripts by inhibiting NMD (often by perturbing UPF1) and using microarray methods (Hansen et al. 2009; Mendell et al. 2004; Pan et al. 2006; Saltzman et al. 2008; Sayani et al. 2008; Wengrod et al. 2013; Wittmann, Hol, and Jäck 2006; Yepiskoposyan et al. 2011). However, microarray analyses generally rely on probes derived from known transcript sequences, again, derived from normal cells where NMD is active. Thus, their capacity to detect native transcripts targeted by NMD is limited, and many 
novel isoforms were likely missed. High-throughput deep sequencing of the transcriptome and current analytical algorithms now allow for the improved identification and quantification of novel isoforms. These methods have been combined with NMD inhibition and used to investigate the effect of NMD on the transcriptome in mouse (Hurt et al. 2013; Weischenfeldt et al. 2012) and in human (Colombo et al. 2017; Lou et al. 2016; Lykke-Andersen et al. 2014; Tani et al. 2012) and deep sequencing of mouse and human brains has revealed extensive alternative splicing coupled to NMD, even in the presence of active NMD (Yan et al. 2015).

Many questions regarding the prevalence and mechanism of NMD remain open. In this study we used NMD inhibition via knockdown of UPF1 and RNA-Seq analysis in a human cell line (HeLa) in order to discover genes that produce putative unproductive isoforms and investigate the features of transcripts affected by NMD. For both known and novel transcripts, we predicted isoform-specific putative PTCs (by the 50nt rule) and measured isoform-level expression changes, in contrast to other studies that used gene expression and annotated coding sequences. Using isoform-level analyses allows us to then filter out genes that are transcriptionally upregulated due to secondary effects of the experiment. Even with this more conservative analysis, we found that a substantial fraction of alternatively spliced transcripts are subject to NMD, even in just this single cell type and condition. Furthermore, we were able to investigate the features of NMD-targeted isoforms and found that the presence of an exon-exon junction $\geq 50 \mathrm{nt}$ downstream of a stop codon was a strong predictor of NMD susceptibility, as expected. However, the length of the 3' UTR on its own had a much smaller effect globally. We also found that NMD targets are enriched for the presence of ultraconserved elements and for splicing regulators and RNA-binding proteins. Additionally, many transcripts with uORFs are sensitive to 
bioRxiv preprint doi: https://doi.org/10.1101/2020.07.01.183327; this version posted July 2, 2020. The copyright holder for this preprint (which was not certified by peer review) is the author/funder, who has granted bioRxiv a license to display the preprint in perpetuity. It is made available under aCC-BY 4.0 International license.

NMD. Our findings include numerous previously unreported substrates for this process in human cells, implying broad regulatory potential. 


\section{Results}

\section{Over two thousand genes produce transcripts identified as high confidence NMD targets}

Transcripts with a premature termination codon (PTC), defined as an early stop codon that triggers NMD, should be stabilized if the NMD pathway is inhibited. To systematically survey NMD-targeted transcripts, we performed strand-specific paired-end RNA-Seq on HeLa cells with inhibited NMD. To inhibit NMD, we used short hairpin RNAs (shRNAs) against the mRNA of NMD factor UPF1 (Bühler et al. 2006; Paillusson et al. 2005). For each of the two biological replicates, a paired control experiment using an shRNA of random sequence was also performed (Bühler et al. 2006). Western blot analysis confirmed that the protein level of UPF1 in the knockdown cells was $6 \%$ of that in the control cells and real-time PCR showed that the abundances of the known NMD-targeted isoforms of SRSF2 and SRSF6 were substantially increased (4-30x), suggesting that NMD was substantially inhibited (Figure S1). The RNA-Seq reads were then aligned to the human reference genome with Tophat (Trapnell, Pachter, and Salzberg 2009) and assembled into known and novel isoforms with Cufflinks (Roberts et al. 2011; Trapnell et al. 2010). After filtering out transcripts with low confidence junctions (see Supplementary Methods), the transcript level abundance was quantified for a set of 131,003 isoforms, 60,976 of which were substantially expressed (Table S1).

We then defined a set of high-confidence direct NMD targets by focusing on those transcripts that showed compelling evidence of being NMD targets, by the 50nt rule model and by our experimental data, as well as by controlling for experimental secondary effects. To do this, we first looked for transcripts with a termination codon more than 50 nucleotides upstream of the transcript's last exon-exon junction. Such a stop codon, which we term a 'PTC50nt', would be a 
putative PTC according to the 50nt rule and is expected to trigger NMD. In order to determine

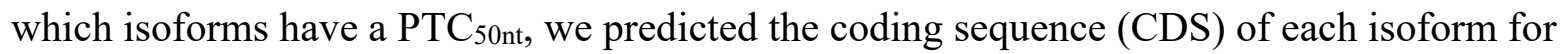
each gene (see Supplementary Methods), resulting in 30,317 expressed CDS-containing isoforms derived from 11,055 genes (Table S1). We found that a fifth $(6,429)$ of these transcripts contained a PTC 50 nt.

Direct targets of NMD should also exhibit significantly higher abundance when NMD was inhibited. We found 10,717 CDS-containing transcripts that increased at least 1.5x and had significant differential expression when NMD was inhibited (Table 1). These were significantly enriched for PTC $_{50 n t}$-containing transcripts (Fisher's exact test, $\mathrm{p}<2.2 \times 10^{-16}$, which is the limit in R). Altogether, 3,832 out of 6,429 $\mathrm{PTC}_{50 \mathrm{nt}}$-containing transcripts $(60 \%)$ were significantly and 1.5x more abundant in NMD inhibited cells, compared to only $871(14 \%)$ that were similarly less abundant (Table 1, Figure 1A). Many other $\mathrm{PTC}_{50 n t}$-containing transcripts demonstrated increased abundance, but were not statistically significant. Confounding transcriptional or splicing changes and uncertainty in the analysis could explain most of the $\mathrm{PTC}_{50 \mathrm{nt}}$-containing transcripts that do not increase. Compared to transcripts with a normally positioned non-PTC $50 \mathrm{nt}$ termination codon (an NTC), PTC $_{50 \mathrm{nt}}$-containing transcripts demonstrated a strong bias towards accumulation when NMD was inhibited (Kolmogorov-Smirov (KS) test, $\mathrm{p}<2.2 \times 10^{-16}$ ). Transcripts with an NTC had similar percentages of significantly increased (29\%) and decreased (28\%) transcripts (Figure 1B), suggesting that they may be affected by secondary effects from the knockdown of the UPF1 protein and are unlikely to have been directly affected by NMD.

Since UPF1 has multiple functions, including those independent of NMD (Kaygun and Marzluff 2005; Kim et al. 2005; Taylor et al. 2013), its depletion could cause gene expression changes at the transcriptional level. Additionally, mis-regulation of genes regulated via 
alternative splicing coupled to NMD could have downstream effects. To control for the potential contribution of these transcriptional changes to the increased abundance of $\mathrm{PTC}_{50 \mathrm{nt}}$-containing transcripts, we further focused on $\mathrm{PTC}_{50 n t}$-containing transcripts that not only were significantly 1.5x more abundant in NMD inhibited cells (and increased in both biological replicates independently), but also adhere to either of the following criteria: 1) no NTC-containing isoform from the same gene was more than 1.2x more abundant in the inhibited NMD sample, or 2) the PTC50nt-containing transcript increased at least $2 \mathrm{x}$ more than did the sum of the NTC-containing isoforms from the same gene. Note that these criteria require that the genes also produce an NTC-containing isoform that is expressed in this experiment. This will exclude genes that are constitutive NMD targets such as some selenoproteins (Moriarty, Reddy, and Maquat 1998; Seyedali and Berry 2014) and some of the NMD factors themselves (Huang et al. 2011; Yepiskoposyan et al. 2011).

We thus identified 2,793 isoforms (from 2,116 genes) as our high confidence set of direct NMD targets (Table S2). These isoforms were derived from 19\% of expressed, protein-coding genes, and $22 \%(2,116$ out of 9,440$)$ of alternatively spliced genes in this cell type (alternatively spliced here defined as producing 2 or more expressed isoforms, including the NMD targets). This set of high-confidence NMD targets makes up over 5\% of all CDS-containing transcripts produced. While amongst these certainly remain a small number of artifacts, we believe a much larger number of true NMD targets are excluded. Before degradation, many of these NMDtargeted transcripts are produced at levels comparable to those of NTC-containing transcripts (Figure S2), and all are expressed at a substantial level, suggesting that they are not merely splicing noise but instead play an important role in regulation. To confirm the expression changes reported by our analysis of the RNA-Seq data, 48 high-confidence NMD-targeted transcripts 
were checked by qPCR and all had $>1.5 x$ increase when NMD was inhibited, consistent with our RNA-Seq results (Figure S3).

\section{Diverse categories of genes are affected by NMD, particularly splicing}

A number of human genes have been previously reported to produce NMD-targeted transcripts, including genes encoding splicing factors and NMD factors. Many of the genes with previously validated NMD-targeted isoforms were found in our results. Specifically, we found that the majority of the genes encoding SR proteins expressed in our data generated an NMDtargeted isoform (10 out of 11, example in Figure 2A), as previously reported (Lareau, Inada, et al. 2007; Ni et al. 2007). The only SR gene without a high-confidence NMD-targeted isoform in our study is SRSF9, which was reported as having a PTC-containing cassette exon based on homology to mouse. A closer investigation showed that there were only a few junction reads supporting the poison cassette exon in our present data, preventing the confident assembly of the isoform. We also found that five NMD factors (UPF2, SMG1, SMG5, SMG6, SMG7) reported to be auto-regulated (Huang et al. 2011; Yepiskoposyan et al. 2011) all showed significant gene expression increases of 1.6x to 3.3x when UPF1 was knocked down (Figure S4), while UPF3A and $U P F 3 B$ showed non-significant gene expression change.

Expanding on the set of splicing-related genes producing NMD-targeted isoforms, we found that a total of 139 genes annotated with the Gene Ontology (GO) term (Ashburner et al. 2000) 'RNA binding' and 73 genes annotated with 'RNA splicing' produce high-confidence NMD targets (Table 2). These GO categories are significantly enriched in NMD-targeted genes (Fisher's exact test, FDR $<0.05$, Figure 3), as is the KEGG pathway 'spliceosome', the SMART domain 'RNA recognition motif', and the Uniprot keywords 'alternative splicing' and 'mrna 
splicng' (DAVID v6.7 (Huang et al. 2007), FDR<0.10, Figure S5). Additionally, the GO categories 'mitochondrion,' 'methyltransferase activity,' and 'metabolic process' are significantly enriched (Figure 3) as are the KEGG pathways 'Aminoacyl-tRNA biosynthesis', and the Uniprot keywords 'mitochondrion', 'transit peptide', and 'polymorphism' (Figure S5). While not significantly enriched, we found that over 4,000 different GO categories contained genes generating NMD-targeted transcripts, indicating NMD-targeted genes are involved in a number of diverse functions such as protein folding, translation, oxidation-reduction processes, vesiclemediated transport, and chromatin modification. Interestingly, six out of the eight genes annotated 'NADPH binding' are high-confidence NMD targets. Categories significantly depleted for genes producing NMD-targeted isoforms include 'extracellular region' and some associated with transcription regulation ('sequence-specific DNA binding', 'regulation of transcription, DNA-dependent', and 'sequence-specific DNA binding transcription factor activity').

We further found that 71 translation-related genes had NMD-targeted transcripts, including many genes encoding ribosomal proteins (32). This is likely an underestimation of the number of ribosomal genes that are targeted by NMD since most ribosome genes are transcriptionally down-regulated in our NMD-inhibited cells (130 of 169 genes annotated 'ribosome' are $>1.2 \mathrm{x}$ down) and this may mask the increased abundance of NMD-targeted isoforms. In total, over a third of expressed ribosomal genes (58 of 169) produced an alternative isoform with a PTC $50 \mathrm{nt}$ and are, therefore, potential NMD targets, even if they do not fall into our high-confidence set.

We then investigated the importance of NMD targets in the gene regulatory networks of 35 human tissues published by GTEx (Ardlie et al. 2015). The networks included $\sim 10,000$ proteincoding genes, of which 6,560 were expressed in the Hela cell lines in our study and 1,214 are high-confidence NMD targets. We compared the centrality of the 1,214 NMD targeted genes to 
the other expressed genes. Centrality measurements (degree, betweenness, and Eigenvector) of NMD targeted genes were not lower than the other genes (Wilcoxon tests, $\mathrm{p}<0.001$ for all tissues); for some tissues, NMD targeted genes even displayed significantly higher level of centralities (Figure S6), indicating an important role for these NMD-targeted genes in human gene regulation.

\section{NMD-targeted genes are enriched for ultraconserved elements}

The splicing events that generate PTCs in the SR genes are associated with highly conserved nucleotide sequences (Lareau, Inada, et al. 2007; Ni et al. 2007), including those termed ultraconserved elements (UCEs). UCEs are regions of 100\% identity between human, mouse, and rat that are at least 200 bp long (Bejerano et al. 2004). To determine if ultraconserved sequences are more generally enriched in genes producing NMD-targeted isoforms, we examined the overlap between 481 reported UCEs and our high-confidence set of NMD targets. We found that 205 UCEs overlapped 125 expressed genes in our data (83 of which overlapped an exon). Of the 2,116 genes producing an NMD-targeted isoform, 26 overlapped an exonic UCE and 12 genes overlapped an intronic UCE. NMD targets are significantly enriched for UCEs overlapping an exon (Fisher's exact test, $\mathrm{p}=6.7 \times 10^{-4}$ ) but not for purely intronic UCEs (Fisher's exact test, $\mathrm{p}=0.41$ ).

Intriguingly, for at least 21 of the 26 genes with exonic UCEs, the UCE covers the alternatively spliced region that generates the PTC, supporting the hypothesis that UCEs may be involved in splicing regulation. Most of these alternative splicing events are cassette exons. Interestingly, we found that the majority of the NMD-targeted genes associated with UCEs encode RNA-binding proteins involved in pre-mRNA processing, but those with other functions 
such as signaling and transcriptional regulation were also found (Table 3). One typical example is MRRF, a mitochondrial ribosome recycling factor involved in ribosome release at translation termination, whose alternative isoform has a multiple cassette exon inclusion event that generates a PTC. The downstream exon and relevant intron overlap 201nt of $100 \%$ conservation between human and rodent (Figure 2B). Some UCEs completely contained within an intron of an NMD-targeted gene may also play a role in regulating splicing to generate PTC-containing isoforms, although the mechanism is less clear.

\section{The 50nt rule is a strong predictor of NMD while a longer 3' UTR has little effect}

While $60 \%$ of transcripts containing a $\mathrm{PTC}_{50 \mathrm{nt}}$ were significantly more abundant when NMD was inhibited (and over $70 \%$ increased at least $1.2 \mathrm{x}$ ), a PTC $\mathrm{PTn}_{50 \mathrm{n}}$ is not believed to be the only trigger of NMD in human cells. The length of the 3' UTR has also been reported to have an effect on whether a transcript is degraded by NMD. To investigate the correlation between downstream exon-exon junctions ('50nt rule') and NMD, we compared the distance between the termination codon and the last exon-exon junction of a transcript with its change in abundance when NMD was inhibited. We found that transcripts with a $\mathrm{PTC}_{50 \mathrm{nt}}$ are twice as likely to increase than those without (Figure S7A), and on average they increase more than $3 x$ while on average those without a $\mathrm{PTC}_{50 \mathrm{nt}}$ do not change (Figures 4A, S9A). Even for transcripts with a short 3' UTR ( $<400 \mathrm{nt})$, we see a strong correlation of NMD with a downstream exon-exon junction (Figure 4A inset). Additionally, there is a clear shift in likelihood of degradation precisely at a distance of 50 nucleotides, as expected by the $50 \mathrm{nt}$ rule.

While we see some correlation between increased 3' UTR length and increased transcript abundance, this is mostly explained by a strong correlation between 3' UTR length and the 
likelihood of a 3' UTR intron. This correlation largely disappears when looking at only transcripts without the 3' UTR intron that would create a PTC 50 nt $($ Figures 4B, S7B, S9B). Without downstream exon-exon junctions, transcripts with a 3' UTR longer than $2000 \mathrm{nt}$ are significantly more likely to increase in abundance when NMD is inhibited than those with 3' UTRs shorter than $400 \mathrm{nt}\left(\mathrm{KS}\right.$ test, $\left.\mathrm{p}=3.84 \times 10^{-10}, \mathrm{D}=0.06\right)$. However, the effect size is small and the average increase for transcripts with a long 3' UTR is only $1.02 \mathrm{x}$ compared to that for transcripts with a $\mathrm{PTC}_{50 \mathrm{nt}}$, which is $3.3 \mathrm{x}$. A PTC 50 nt has a strong effect even for transcripts with a 3'UTR less than $400 \mathrm{nt}$ long (2.3x average increase; KS test, $\left.\mathrm{p}<2.2 \times 10^{-16}, \mathrm{D}=0.23\right)$. We also investigated the effects of CDS length and GC content of the 3' UTR. For NTC-containing isoforms, ones with a CDS longer than $2000 \mathrm{nt}$ are significantly more likely to increase in abundance than those with a CDS shorter than $400 \mathrm{nt}\left(\mathrm{KS}\right.$ test, $\left.\mathrm{p}<1.58 \times 10^{-7}, \mathrm{D}=0.07\right)$ (Figures S7C, S8A, S9C). Similarly, NTC-containing isoforms with 3' UTR GC content higher than $55 \%$ are more likely to increase than those with GC content less than $35 \%$ (KS test, $\mathrm{p}<2.2$ x $\left.10^{-16}, \mathrm{D}=0.18\right)$ (Figures S7D, S8B, S9D).

\section{Premature termination codons can be generated through alternative splicing events or translation of uORFs}

We also explored the types of molecular events that can generate a putative PTC, including alternative splicing and upstream open reading frames (uORFs). Since all of the transcripts in our high-confidence set of NMD targets come from genes that also produce NTC-containing isoforms, we infer that the PTCs were generated by an alternative splicing event. Using the JuncBASE program (Brooks et al. 2011) to investigate these alternative splicing events, we found that at least 365 of the isoforms $(13 \%$, of 2,793$)$ were generated by the introduction of a PTC-containing cassette exon (poisonous exon) and 54 isoforms had a retained intron containing 
a PTC. Additionally, 257 NMD-targeted isoforms (9\%) were generated by the introduction of an intron downstream of the termination codon of the productive coding sequence. One interesting example of this is $K H D R B S 1$, encoding a protein involved in both signal transduction and mRNA processing, for which alternative splicing combined with alternative poly-adenylation results in the formation of an NMD target without affecting the structure of the coding sequence (Figure 2C).

NMD may also act on transcripts with translated uORFs since the uORF termination codon will almost always be recognized as premature, provided that the main coding sequence is not also translated via translation re-initiation. We examined NTC-containing transcripts for uORFs and found that 14,348 NTC-containing transcripts had at least one uORF, but only 5,628 of these had a uORF with a strong Kozak signal sequence for translation initiation (Kozak 1991). Transcripts with a 'strong' uORF that either overlaps the main CDS or is at least 35 amino acids long are about twice as likely to significantly increase in abundance when NMD is inhibited, compared to those with no uORF (Table S3), similar to what has been reported for plants (Nyikó et al. 2009). Transcripts with a shorter 'strong' uORF are also more likely to increase, as are those with 'weak' uORFs. Thus, our data suggests that hundreds to thousands of transcripts with uORFs are naturally targets of NMD and could be regulated through the potential translation of uORFs. 


\section{Discussion}

In this study, through depletion of UPF1 (a key regulator in the NMD pathway) in HeLa cells, we stabilized transcripts that are otherwise degraded by NMD and determined their structure and expression via RNA-Seq analysis. We used an isoform-centric approach in order to establish a high-confidence set of direct NMD targets and genes that have the potential to be regulated via alternative splicing coupled to NMD. For each transcript, we predicted the coding sequence and did not rely on annotated termination codons, which may be inaccurate and do not exist for novel transcripts. For this analysis, we defined PTCs based on the 50nt rule (Nagy and Maquat 1998) since other features that can trigger NMD have been shown to have low predictive value in our study and others (Colombo et al. 2017; Lindeboom, Supek, and Lehner 2016). While other similar studies use gene-level expression changes to find putative NMD targets (Colombo et al. 2017; Tani et al. 2012), we used isoform-level expression despite the added uncertainty of assigning reads that map to constitutive sequences to the correct isoforms.

Crucially, this allowed us to control for transcriptional increases due to secondary effects, which we believe to be very prevalent in the experiment given the large fraction of transcripts that decrease in abundance. This analysis is conservative and likely to miss NMD targets expressed at a lower level or which did not meet our strict requirements.

We report a set of 2,116 genes as high-confidence NMD targets (19\% of genes with at least one expressed isoform). The high abundance of these transcripts in the NMD-inhibited sample indicates that they are unlikely to be produced by random errors (Figure S2). However, we cannot know what regulatory role they may have and cannot rule out the possibility that they are nonfunctional transcripts with little or no selective impact. Our results re-identified most wellknown NMD targets particularly those derived from the SR genes (Lareau, Inada, et al. 2007; Ni 
et al. 2007), and discovered many more derived from other genes, including many splicing factors. Numerous genes involved in a variety of other functional categories including aminoacyl-tRNA biosynthesis, vesicle-mediated transport, chromatin modification, and metabolic pathways were also found to produce NMD-targeted transcripts, suggesting that a broad range of biological processes may be affected by NMD (Figure 3). Transcripts degraded by NMD are significantly enriched for ultraconserved elements of the mammalian genome, supporting a potential role for these elements in regulation through alternative splicing-coupled NMD. Ultimately, we extrapolate that $20 \%$ or more of human genes produce NMD-targeted alternative isoforms and are potentially post-transcriptionally regulated by NMD coupled with alternative splicing.

Many NMD-targeted genes, especially splicing factors, are thought to be auto-regulated through a feedback loop wherein protein abundance affects the relative levels of productive and unproductive transcripts. The model is that the protein abundance directly or indirectly affects splicing of its own pre-mRNA so that when a protein is present at an unnecessarily high level, the splicing apparatus shifts to generating NMD-targeted isoforms. We found that when NMD is inhibited, over 500 NMD-targeted genes have decreased expression of NTC-containing isoforms, apparently shifting splicing toward generating the NMD-targeted isoform and likely results in the lower abundance of those proteins. This group of genes includes many involved in RNA splicing. In fact, over half (18/31) of the NMD-targeted genes with the GO annotation 'RNA processing' fall into this group, as do all 6 genes annotated as 'heterogeneous nuclear ribonucleoprotein complex.' We also found 180 NMD-targeted genes with decreased expression at both the transcriptional level and the NTC-containing isoform level. These are enriched for genes involved in the ribosome and translation, and their down-regulated expression might be a side 
effect of the UPF1 knockdown, which results in slowed cell growth compared to control cells. Such down-regulation may be mediated through the concerted effort of transcriptional regulation and alternative splicing coupled with NMD. Thus, hundreds of genes were found with evidence of possible regulation through NMD, indicating such a regulatory system may be much more prevalent than previous thought.

The 50nt rule was the first model proposed to be the general signal for NMD degradation in mammals (Nagy and Maquat 1998), and remains the most prevalent model (most cited) with substantial experimental support. However, a longer 3'-UTR has been proposed to also play a role (Hogg and Goff 2010; Hurt et al. 2013; Yepiskoposyan et al. 2011). Results indicating that a long 3' UTR is enough to cause NMD have come from experiments done with a particular construct (Hogg and Goff 2010), or by looking at the annotated 3' UTR length of genes or transcripts that increase in overall abundance when NMD is inhibited (Hurt et al. 2013; Yepiskoposyan et al. 2011). In our present data, we identify the precise isoforms that are degraded by NMD in normal cells, thereby allowing for the discovery of novel instances of $\mathrm{PTC}_{50 \mathrm{nt}}$ as well as directly measuring the 3' UTR length of a specific isoform. Strikingly, the average isoform abundance change when NMD is inhibited is a $3.3 \mathrm{x}$ increase for $\mathrm{PTC}_{50 \mathrm{nt}^{-}}$ containing transcripts, compared to no change on average for NTC-containing transcripts. Since we find that the majority of $\mathrm{PTC}_{50 \mathrm{nt}}$ S fall within the main coding region, those transcripts are going to have longer 3' UTRs, but a long 3' UTR in the absence of a PTC $_{50 n t}$ has a much smaller effect on isoform abundance. Similar results were recently reported after examining transcript abundance across the different polysome fractions, which can indicate translation and NMD status (Lloyd, et al. 2020). One explanation for this lack of a strong effect by 3'UTR length on 
average is that elements in the 3' UTR near the termination codon have been reported to protect against NMD (Ge et al. 2016; Kishor, Ge, et al. 2019; Toma et al. 2015).

Thirty percent of transcripts with a $\mathrm{PTC}_{50 \mathrm{nt}}$ do not increase at least $1.2 \mathrm{x}$ when NMD is inhibited. However, it is possible that as few as 5\% are actually escaping NMD. For the others, we see evidence of transcriptional or splicing regulation that could mask the stabilization of the transcript (including transcriptional down-regulation or a shift in splicing toward the productive isoform) and/or aspects that lead to increased uncertainty in the analysis (such as low sequencing coverage, incorrect transcript assembly or CDS definition, and complex alternative splicing patterns). PTC $50 \mathrm{nt}$-containing transcripts can escape NMD through secondary structure in the 3' UTR (Eberle et al. 2008), alternative poly-adenylation sites (Gilat and Shweiki 2007), or differential deposition of the exon junction complex required for NMD (Alexandrov et al. 2012; Saulière et al. 2010), as well as other mechanisms (Dyle et al. 2019; Hoek et al. 2019; Kishor, Fritz, et al. 2019; Neu-Yilik et al. 2011; Peixeiro et al. 2012). We conclude that while 3' UTR length may have an effect for a subset of genes, the 50nt rule is the major mechanism of targeting a transcript for NMD in humans with the caveat that our work is on 'natural' NMD targets and it is unclear what happens in 'unnatural' mutation cases.

Based on the above findings, we would not expect most transcripts without a PTC $_{50 \text { nt }}$ to be degraded by NMD. While we find that almost $30 \%$ of NTC-containing transcripts significantly increase when NMD is inhibited, almost the same number decrease in abundance, leading us to believe that these changes are likely due to secondary effects of the knockdown experiment. We sought features of these increasing transcripts that may indicate they are targeted for NMD. As described above, we find little evidence that 3' UTR length significantly affects likelihood of degradation. The length of the coding sequence also does not have a strong effect (Figure S8A). 
We see a correlation between 3' UTR GC content and likelihood of increased abundance consistent with evidence that UPF1 binds G-rich sequences (Hurt et al. 2013) and that GC motifs in the 3'UTR are important for NMD (Imamachi et al. 2016). Finally, we found that for thousands of transcripts, having a uORF with a strong Kozak signal sequence almost doubles the likelihood that they increase in abundance in UPF1-depleted cells, indicating that NMD may also be involved in extensive regulation of gene expression via a mechanism that is sensitive to the translation of uORFs as has been reported for the ATF5 gene (Hatano et al. 2013). uORF transcripts are also often bound by a single ribosome, suggesting a low translation rate, and is consistent with many being NMD targets (Lloyd et al. 2020).

In conclusion, alternative splicing coupled to NMD has the potential to massively shape the transcriptome of human cells by directly regulating at least $20 \%$ of alternatively spliced genes as well as being a crucial component of splice factor regulation, which itself has numerous downstream regulatory effects. Uncertainty in short-read RNA-Seq analysis may result in some false positives in our NMD target set, however, our conservative approach in defining our highconfidence set means there are likely far more targets that are being missed (false negatives). While this analysis was performed for only one cell line, HeLa, which may have nonphysiological events, it is likely the number of NMD targets will increase when investigating other cell types. Since NMD-targeted transcripts are often found at very low levels or not at all in normal cells, this type of regulation is often overlooked when studying a gene or pathway. However, the potential regulatory scope of alternative splicing coupled to NMD demonstrates that researchers should take care to take this regulation into account. 


\section{Materials and Methods}

\section{Knockdown of UPF1 by shRNA}

HeLa cells were inoculated on plates in Dulbecco's MEM medium with $0.1 \mathrm{mM}$ non-essential amino acids and $10 \%$ fetal bovine serum, incubated at $37^{\circ} \mathrm{C}$ and $5 \% \mathrm{CO}_{2}$. Plates at $80 \%$ cell confluency were transfected with a mixture of the plasmids pSUPERpuro-hUpf1 I and II or with pSUPERpuro-Scramble (a gift from Oliver Mühlemann's lab (Bühler et al. 2006; Paillusson et al. 2005)), whose functions were to knockdown UPF1 and act as a mock control, respectively. Transfections were performed using Lipofectamine ${ }^{\mathrm{TM}}$ LTX and PLUS ${ }^{\mathrm{TM}}$ Reagents (Invitrogen) according to the manufacturer's protocol, and the following culture according to the published method (Paillusson et al. 2005). The whole cell lysates were prepared in 1\% sodium dodecyl sulfate and incubated at $100{ }^{\circ} \mathrm{C}$ for $5 \mathrm{~min}$, then centrifuged at $12,000 \mathrm{rpm}$ for $10 \mathrm{~min}$. The extracted total protein was quantified using the micro $\mathrm{BCA}^{\mathrm{TM}}$ protein assay kit (Pierce Company). Knockdown efficiency was validated by Western blot analysis, using $\beta$-actin as the control. Odyssey ${ }^{\circledR}$ infrared imaging system (Li-Cor) was used to quantify and compare the UPF1 protein level between UPF1-knockdown and control samples. Total RNA was extracted using the QIAGEN RNeasy ${ }^{\circledR}$ Mini kit according to the manufacturer's manual. RNA concentration was determined by NanoDrop $2000^{\circledR}$, and RNA integrity was determined by $1.2 \%$ agar gel and BioAnalyzer. NMD inhibition was validated by amplification of two known NMD-degraded transcripts using oligos from (Lareau, Inada, et al. 2007) by real-time PCR with ABI 7500 fast real time PCR system (Applied Biosystems), and data were analyzed using 7500 software v2.04 (Applied Biosystems). 


\section{Preparation of RNA-Seq libraries}

Directional and paired-end RNA-Seq libraries were constructed according to the Illumina protocol, with a few changes: The adapters were prepared according to the reported methods (Vigneault, Sismour, and Church 2008). The PCR process to prepare the library was divided in two steps. In the first step, 3 cycles of PCR were performed (according to the protocol) to prepare the library template, and then the library was run on a $2 \%$ agarose gel, fragments of desired size were cut out and isolated by QIAquick ${ }^{\circledR}$ Gel Extraction Kit. A second round of PCR (12 cycles) was performed to enrich the library and then it was purified twice with Agencourt AMPure XP kit as suggested by the Illumina protocol. The libraries were then assayed on a Agilent 2100 BioAnalyzer. These RNA-Seq libraries were prepared from cells with inhibited NMD and control cells for two biological replicates. One biological replicate was sequenced on an Illumina GAIIx machine with $80 \mathrm{bp}$ reads (40 million paired-end reads produced) and the other on HiSeq 2000 with 100 bp reads (180 million paired-end reads produced).

\section{Transcript assembly and abundance quantification}

Paired end reads for each library were aligned to the NCBI human RefSeq transcriptome (Pruitt et al. 2009) with Bowtie (Langmead et al. 2009) to determine the average insert size and standard deviation, required as a parameter by TopHat (Trapnell et al. 2009). The reads of each library were then aligned to the human genome (hg19 assembly, Feb. 2009) using TopHat v1.2.0 with default parameters plus the following: --solexa1.3-quals, --library-type fr-secondstrand, -coverage-search, --allow-indels, --microexon-search, and --butterfly-search (over $80 \%$ of reads aligned). Cufflinks v1.0.1 (Roberts et al. 2011; Trapnell et al. 2010) was used to assemble each set of aligned reads into transcripts with the UCSC known transcript set (downloaded Jan. 2011 
from http://genome.ucsc.edu/ (Fujita et al. 2011)) as the reference guide, along with the following parameters: --frag-bias-correct, and --multi-read-correct. Cuffcompare (a sub-tool of Cufflinks) was used to merge the resulting sets of assembled transcripts. Each junction was assigned a Shannon entropy score based on offset and depth of spliced reads across all four libraries (Brooks et al. 2011), see Supplementary Methods for details. Transcripts with a junction that had an entropy score $<1$ and were not present in the reference annotation were filtered out. Cuffdiff (a sub-tool of Cufflinks) was used to quantify and compare transcript abundance (measured by FPKM, Fragments Per Kilobase per Million reads) between the UPF1 knockdown and control samples. For each sample, the reads from two biological replicates were provided. The following parameters were used: --frag-bias-correct and --multi-read-correct. Only transcripts with FPKM $>1$ in either the control or UPF1 knockdown sample were used for further analysis. A transcript was called significantly more abundant in the UPF1 knockdown sample if Cuffdiff called it significantly changing and the change was greater than $1.5 \mathrm{x}$.

Significantly decreased transcript abundances were determined in the same way (change was greater than $1.5 \mathrm{x})$.

\section{Determination of NMD targets}

For each transcript, the coding sequence (CDS) was determined as described in the Supplementary Methods, based on (Hansen et al. 2009). A coding sequence was defined to terminate in a premature stop codon $\left(\mathrm{PTC}_{50 \mathrm{nt}}\right)$ if its termination codon was at least 50 nucleotides upstream of the last exon-exon junction of the transcript (50nt rule in mammals). We inferred transcripts to be NMD targets if they met several criteria. The transcripts must have both a $\mathrm{PTC}_{50 \mathrm{nt}}$ and significantly and $>1.5 \mathrm{x}$ increased abundance in NMD inhibited (UPF1 knockdown) cells. The transcripts must also increase in each biological replicate when analyzed 
independently and come from a gene with a NTC-containing isoform with FPKM $>0$. A subset of these were deemed more reliable, which we termed our high-confidence NMD-targeted transcripts. High-confidence NMD targeted transcripts adhered to either of the following criteria were kept: 1) No NTC-containing isoform from the gene was more than 1.2x higher in the NMD inhibited sample, or 2) the PTC $50 \mathrm{nt}$-containing isoform increased at least $2 \mathrm{x}$ more than the sum of all NTC-containing isoform FPKMs from the gene in NMD inhibited cells.

Functional classification was based on Ensemble gene annotation, using Gene Ontology (GO) terms (Ashburner et al. 2000), downloaded from Ensembl BioMart Nov 2011. Functional enrichment or depletion was determined by a one-tailed Fisher's Exact test for NMD targeted genes against the background of all genes with an expressed CDS-containing isoform (FPKM > 1 in at least one sample). A False Discovery Rate threshold of 0.05 was used to correct for multiple tests $(4,328$ tests done for enrichment (GO categories with at least one NMD target), and 9,673 tests for depletion (all GO categories)). Alternative splicing events were characterized with JuncBASE (version 120623) (Brooks et al. 2011). The JuncBASE pipeline was run on each of the two biological replicates independently with default parameters. The Cuffcompare output GTF, filtered for unsupported junctions, was used as the database for defining exons. A strong Kozak signal for a $\mathrm{uORF}$ start codon was defined as $[\mathrm{A} / \mathrm{G}] \mathrm{NNaugG}[$ not $\mathrm{U}]$ as recommended by the Consensus CDS Project (Harte et al. 2012).

\section{Validation by real-time PCR}

To validate the potential NMD-targeted transcripts found in the present study, we measured their relative mRNA abundance in cells with UPF1 knocked down and control cells using quantitative real-time PCR. Total RNAs were extracted as described above. cDNAs were synthesized from 5 
$\mu \mathrm{g}$ total RNA using the SuperScript II first-strand cDNA synthesis system (Invitrogen) according to the manufacturer's instructions. The isoform-specific primers used are listed in Table S4 (those for SRSF genes were from (Lareau, Inada, et al. 2007)). Real-time PCRs were performed on Applied Biosystems 7500 Fast Real-Time PCR System using the respective pair of primers designed with Primer Premier 6.0 and Maxima SYBR Green/ROX qPCR Master Mix (Thermo Scientific). PCR reactions were performed in four replicates, and the expression levels were normalized to that of $\beta$-actin control gene (NM_001101).

\section{Data availability}

The raw reads are deposited in the NCBI SRA database under the BioProject Accession ID PRJNA294972 (SRA Accession ID SRP063462). Datasets used in this study are available for download at dash.berkeley.edu available at: https://doi.org/10.6078/D1H019 


\section{Acknowledgements}

We thank Adam Roberts and Lior Pachter for help with the optimization of Cufflinks. This work was funded by NIH Grant R01 GM071655 to SEB, GW was supported by a Tang Distinguished Scholarship from QB3 at UC Berkeley, CEF was supported by NIH T32 GM007232 and T32

HG000047 training grants, and by the Department of Defense (DoD) through the National Defense Science \& Engineering Graduate Fellowship (NDSEG) Program, and JPBL was supported by the Center for RNA Systems Biology (NIH Grant P50 GM102706 to Jamie Cate). 
bioRxiv preprint doi: https://doi.org/10.1101/2020 07.01.183327; this version posted July 2, 2020. The copyright holder for this preprint (which

was not certified by peer review) is the author/funder, who has granted bioRxiv a license to display the preprint in perpetuity. It is made available under aCC-BY 4.0 International license.

\section{Author contributions:}

CEF: designed and performed analysis and wrote manuscript

GW: designed and performed experiments

JPBL: reviewed and edited manuscript

$\mathrm{ZH}$ : performed analysis

ANB: designed analysis

SEB: designed experiment and analysis, reviewed and edited manuscript.

SEB reviewed the penultimate version but not the final submitted version due to an accident injury. 


\section{Tables}

Table 1. Classification of expressed transcripts with and without a PTC50nt

\begin{tabular}{|l|c|c|c|}
\hline & $\begin{array}{c}\text { Number of } \\
\text { isoforms }\end{array}$ & $\begin{array}{c}\text { Increased } \\
\text { abundance }\end{array}$ & $\begin{array}{c}\text { Decreased } \\
\text { abundance }^{\dagger}\end{array}$ \\
\hline $\begin{array}{l}\text { All expressed transcripts with } \\
\text { a defined coding sequence }\end{array}$ & 30,317 & $\begin{array}{l}10,717 \\
(35 \%)\end{array}$ & $\begin{array}{c}7,600 \\
(25 \%)\end{array}$ \\
\hline Transcripts with a NTC & 23,888 & $\begin{array}{c}6,885 \\
(29 \%)\end{array}$ & $\begin{array}{c}6,729 \\
(28 \%)\end{array}$ \\
\hline Transcripts with a PTC50nt & 6,429 & $\begin{array}{c}3,832 \\
(60 \%)\end{array}$ & $\begin{array}{c}871 \\
(14 \%)\end{array}$ \\
\hline
\end{tabular}

'Increased and decreased abundance refers to the expression changes in NMD-inhibited cells (compared to control cells) that are significant according to Cuffdiff and $>1.5 \mathrm{x}$. The percentage is of the number of transcripts in that category.

Table 2. Genes generating an isoform degraded by NMD are enriched for those involved in RNA splicing

\begin{tabular}{|c|c|}
\hline $\begin{array}{l}\text { Splice factor } \\
\text { category }\end{array}$ & $\begin{array}{l}\text { Genes annotated with the GO term 'RNA splicing' with isoforms degraded by NMD } \\
\text { (underlined genes were previously found to have NMD-targeted isoforms) }\end{array}$ \\
\hline SR & $\underline{\text { SRSF1}}, \underline{\text { SRSF2 }}, \underline{\text { SRSF3 }}, \underline{\text { SRSF4 }}, \underline{\text { SRSF5 }}, \underline{\text { SRSF6 }}, \underline{\text { SRSF7 }}, \underline{\text { SRSF8 }}, \underline{\text { SRSF10 }}, \underline{\text { SRSF11 }}$ \\
\hline hnRNP & 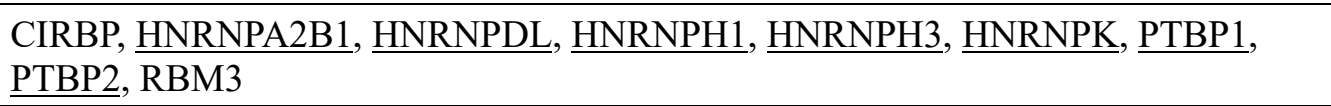 \\
\hline snRNP & PPIH, PRPF3, SART1, SNRNP40, SNRNP48, SNRNP70, TXNL4A, U2AF1, U2AF2 \\
\hline DEAD & DDX5, DDX46, DHX9, DHX15, DHX35, INTS6 \\
\hline $\mathrm{Sm}$ & SNRPA1, SNRPB, SNRPN \\
\hline Other & $\begin{array}{l}\text { ACIN1, AKAP17A, CCAR1, C16orf80, CDK12, CIR1, CLASRP, CLK1, CPSF1, } \\
\text { CRNKL1, CSTF1, DGCR14, DNAJC8, EIF2S2, FUBP3, FUS, GCFC1, GTF2F2, } \\
\text { IVNS1ABP, LUC7L3, MAGOHB, MOV10, NCBP2, POLR2E, PRPF4, PRPF4B, } \\
\text { PRPF18, PRPF38B, RBM25, SCAF8, SF3B1, SFPQ, SFSWAP, SMNDC1, SRPK1, } \\
\text { SREK1, SRRM1, SRRM2, SUGP1, SUGP2, TCERG1, THOC2, THOC4, TIA1, } \\
\text { TIAL1, TOP1MT, TRA2A, TRA2B, TXNL4B, U2SURP, USP39, YTHDC1, } \\
\text { ZCCHC8, ZFR, ZNF207, ZRSR2 }\end{array}$ \\
\hline
\end{tabular}


bioRxiv preprint doi: https://doi.org/10.1101/2020.07 01.183327; this version posted July 2, 2020. The copyright holder for this preprint (which

was not certified by peer review) is the author/funder, who has granted bioRxiv a license to display the preprint in perpetuity. It is made available under aCC-BY 4.0 International license.

Table 3. Genes with NMD-targeted isoforms are enriched for ultraconserved elements

Functional category

RNA processing

Transcriptional regulation

Other
Genes where the UCE overlaps the alternative splicing event that generates the NMD-targeted isoform are underlined

DDX5, DHX15, HNRNPH1, HNRNPK, HNRPDL, PRPF38B,

$16 \underline{\text { PTBP2 }}, \underline{\text { SRSF1, }} \underline{\text { SRSF3 }}, \underline{\text { SRSF6 }}, \underline{\text { SRSF7, }}$ SRSF11, TIAL1, TRA2A, TRA2B, ZFR

4 CCAR1, MED1, $\underline{\text { MGA, }}$ NFAT5

6 HIRA, RC3H2, $\underline{\text { FAM98A, }}$ MRRF, $\underline{\text { STRN3 }}, \underline{\text { DLG2 }}$ 


\section{Figure 1.}

A.

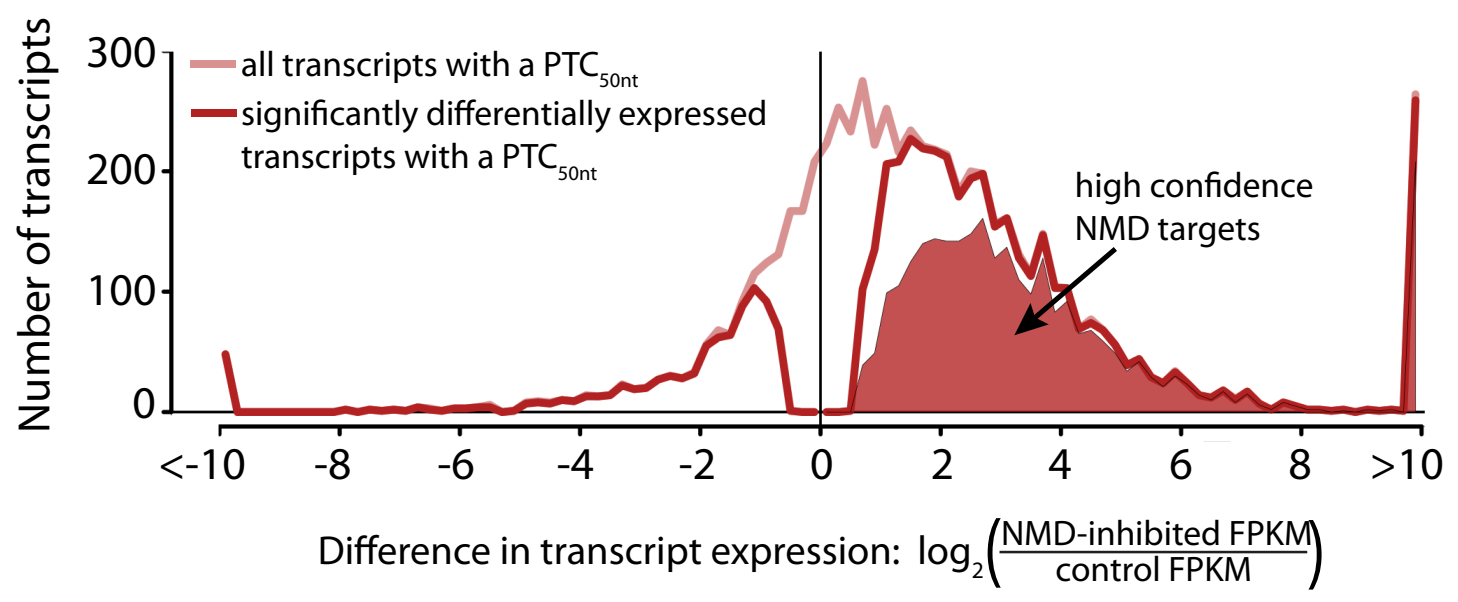

B.

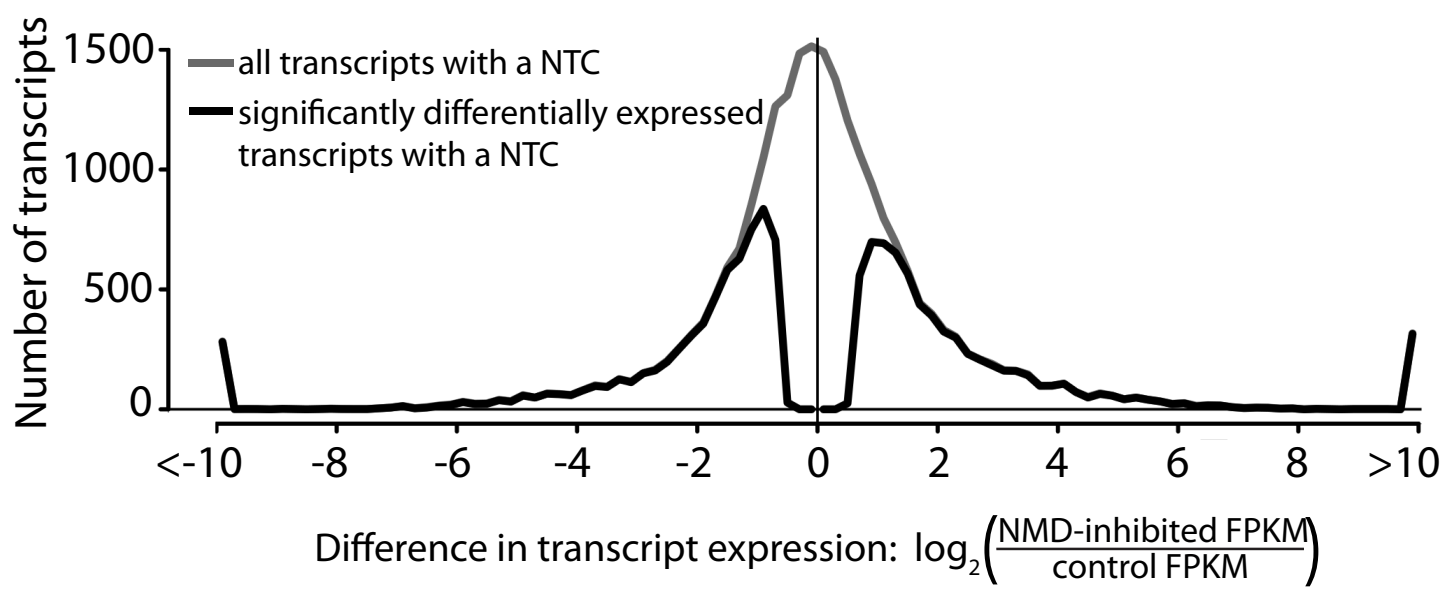




\section{Figure 2.}

\section{A. SRSF3}

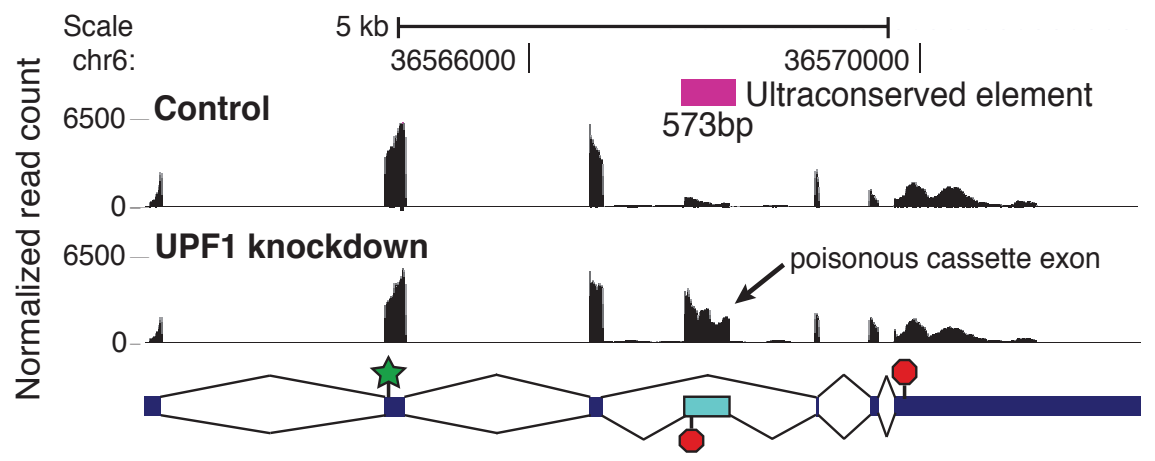

\section{B. MRRF}

\section{Scale}

chr9: $125,040,000$ ।

‡ 665 Control

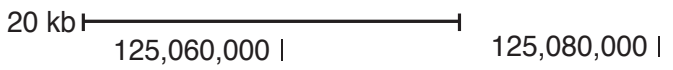

$5,060,000$

○े

자 0

665 UPF1 knockdown

월

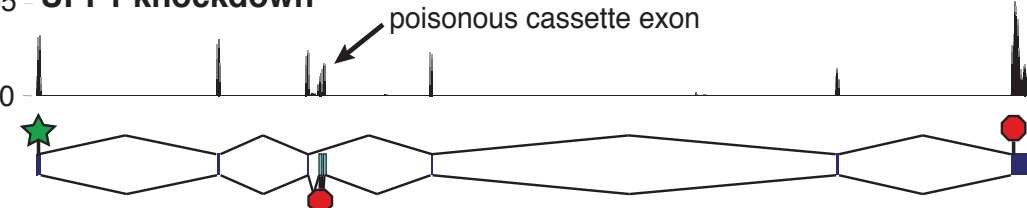

Ultraconserved element 203bp

\section{KHDRBS1}

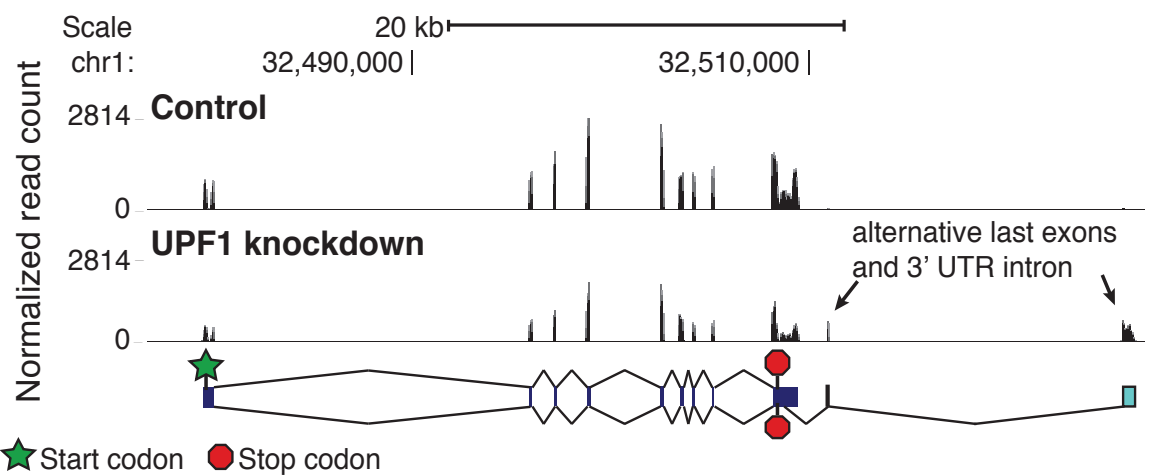




\section{Figure 3.}

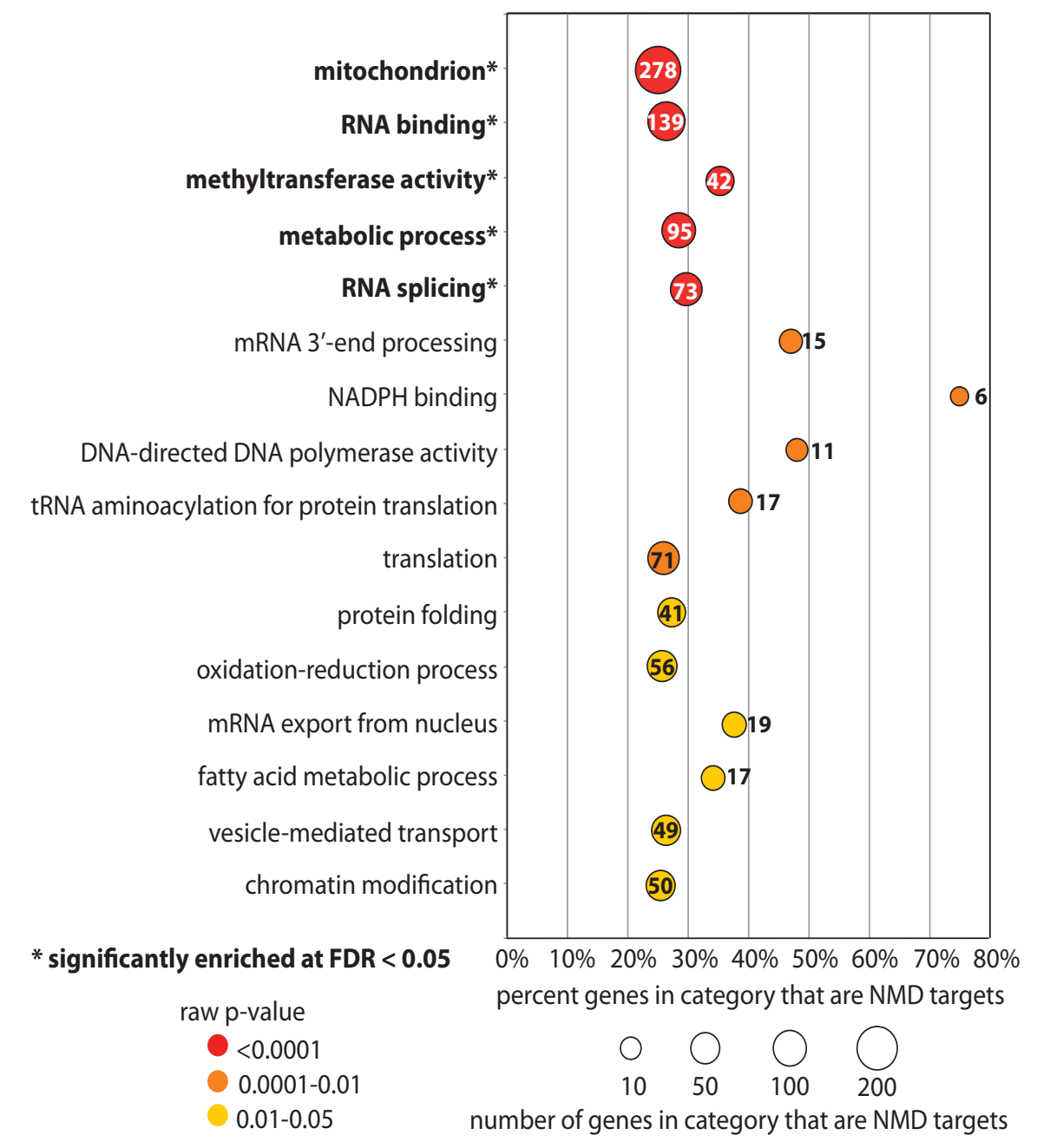




\section{Figure 4.}

A.

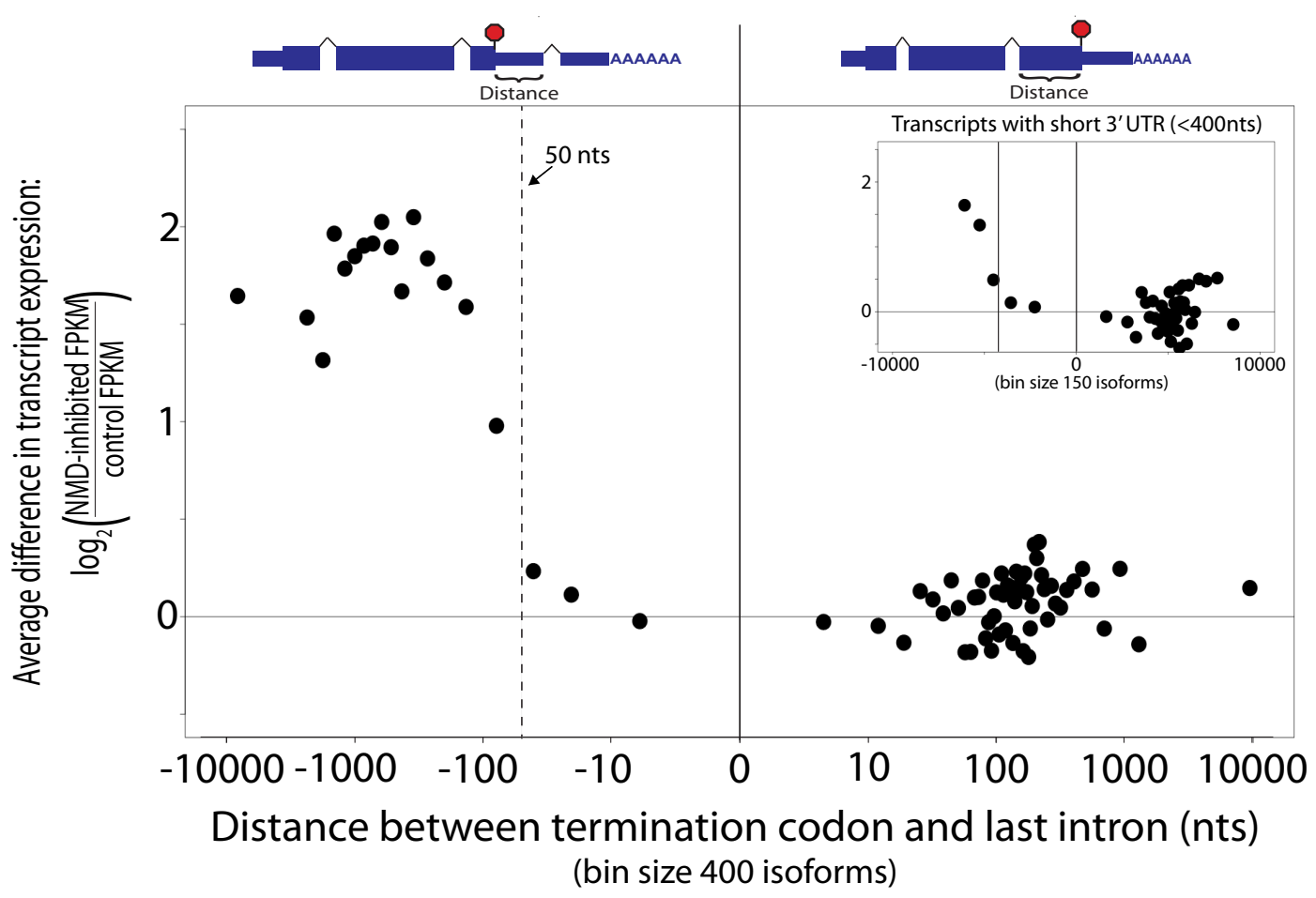

B.

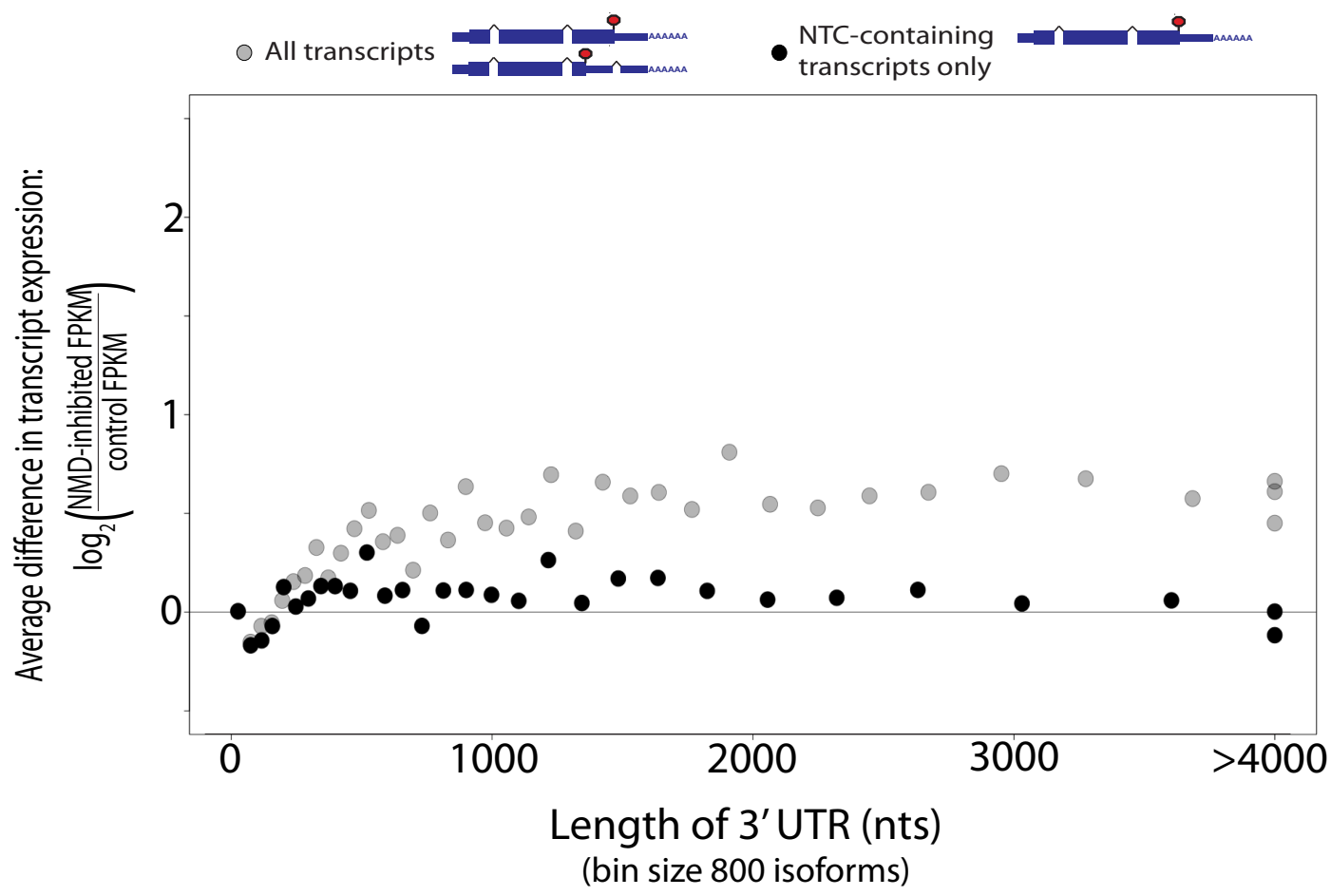




\section{Figure legends}

Figure 1. Transcripts with a $\mathrm{PTC}_{50 \mathrm{nt}}$ are more likely to increase in abundance when NMD is inhibited.

A) Histogram of expression changes of PTC $_{50 n t}$-containing transcripts between NMD inhibited cells and control cells. B) Histogram of expression changes of NTC-containing transcripts between NMD inhibited cells and control cells. Transcripts that were not expressed in one of the conditions were given a $\log 2$ (inhibited $\mathrm{FPKM} /$ control $\mathrm{FPKM}$ ) value of $\pm 10 . \mathrm{PTC}_{50 \mathrm{nt}}$-containing transcripts demonstrated a strong bias towards accumulation when NMD was inhibited (Kolmogorov-Smirov (KS) test, $\mathrm{p}<2.2 \times 10-16$ ).

Figure 2. Previously inferred splicing events resulting in NMD targets were confirmed and new ones were discovered (SRSF3, MRRF, KHDRBS1).

A) The splicing factor SRSF3 is a previously known NMD target with an ultraconserved element overlapping the $\mathrm{PTC}_{50 \mathrm{nt}}+$ exon. B) MRRF, a mitochondrial ribosome recycling factor, is an example of a non-splicing factor gene with an NMD-targeted transcript associated with an ultraconserved element. The element overlaps the 3' splice site of the alternative splicing event that generates the $\mathrm{PTC}_{50 \mathrm{nt}}$. C) KHDRBS1, encoding a protein involved in both signal transduction and mRNA processing, has an NMD-targeted transcript generated by alternative splicing and poly-adenylation downstream of the normal termination codon. This figure was generated with the UCSC Genome Brower. Normalized read counts (\# reads/10 million mapped reads) for control cells (top track) and NMD-inhibited cells (bottom track) were plotted. Isoform structures are shown below the read tracks. The stop indicated underneath the exon is the 
PTC $_{50 n t}$. A magenta box above the read tracks indicates the presence of an ultraconserved element.

Figure 3. Genes generating an isoform degraded by NMD fall into diverse functional categories.

Genes with an isoform degraded by NMD were classified into Gene Ontology functional categories. The asterisk indicates the category is significantly enriched for genes with NMDtargeted isoforms (Fisher's exact test FDR $<0.05$; background is genes with an expressed isoform (FPKM $>1$ in at least one sample)). The test was performed with the fisher.test function in $\mathrm{R}$, the multiple hypothesis correction done in Excel and the plot with ggplot and Adobe Illustrator).

Figure 4. The 50nt rule is a strong predictor of NMD while a longer 3' UTR is not.

A) The distance the termination codon is downstream of the last exon-exon junction versus $\log 2$ (inhibited FPKM/control FPKM). Each point is the average log2 (inhibited FPKM/control FPKM) vs the median distance of 400 isoforms. B) Length of the 3' UTR versus log2 (inhibited FPKM/control FPKM). Gray: all expressed transcripts. Black: Only expressed NTC-containing transcripts. Each point is the average log2 (inhibited FPKM/control FPKM) vs the median distance of 800 isoforms. Bins with median 3' UTR length more than 4000 nts were placed at 4000 nts. Plots were generated with R. 


\section{References}

Alexandrov, A., D. Colognori, M. D. Shu, and J. A. Steitz. 2012. "Human Spliceosomal Protein CWC22 Plays a Role in Coupling Splicing to Exon Junction Complex Deposition and Nonsense-Mediated Decay." Proceedings of the National Academy of Sciences.

Amrani, Nadia, Robin Ganesan, Stephanie Kervestin, David A. Mangus, Shubhendu Ghosh, and Allan Jacobson. 2004. "A Faux 3'-UTR Promotes Aberrant Termination and Triggers Nonsense-Mediated MRNA Decay." Nature.

Änkö, Minna Liisa, Michaela Müller-McNicoll, Holger Brandl, Tomaz Curk, Crtomir Gorup, Ian Henry, Jernej Ule, and Karla M. Neugebauer. 2012. "The RNA-Binding Landscapes of Two SR Proteins Reveal Unique Functions and Binding to Diverse RNA Classes." Genome Biology.

Ardlie, Kristin G., David S. DeLuca, Ayellet V. Segrè, Timothy J. Sullivan, Taylor R. Young, Ellen T. Gelfand, Casandra A. Trowbridge, Julian B. Maller, Taru Tukiainen, Monkol Lek, Lucas D. Ward, Pouya Kheradpour, Benjamin Iriarte, Yan Meng, Cameron D. Palmer, Tõnu Esko, Wendy Winckler, Joel N. Hirschhorn, Manolis Kellis, Daniel G. MacArthur, Gad Getz, Andrey A. Shabalin, Gen Li, Yi Hui Zhou, Andrew B. Nobel, Ivan Rusyn, Fred A. Wright, Tuuli Lappalainen, Pedro G. Ferreira, Halit Ongen, Manuel A. Rivas, Alexis Battle, Sara Mostafavi, Jean Monlong, Michael Sammeth, Marta Melé, Ferran Reverter, Jakob M. Goldmann, Daphne Koller, Roderic Guigó, Mark I. McCarthy, Emmanouil T. Dermitzakis, Eric R. Gamazon, Hae Kyung Im, Anuar Konkashbaev, Dan L. Nicolae, Nancy J. Cox, Timothée Flutre, Xiaoquan Wen, Matthew Stephens, Jonathan K. Pritchard, Zhidong Tu, Bin Zhang, Tao Huang, Quan Long, Luan Lin, Jialiang Yang, Jun Zhu, Jun Liu, Amanda Brown, Bernadette Mestichelli, Denee Tidwell, Edmund Lo, Michael Salvatore, Saboor Shad, Jeffrey A. Thomas, John T. Lonsdale, Michael T. Moser, Bryan M. Gillard, Ellen Karasik, Kimberly Ramsey, Christopher Choi, Barbara A. Foster, John Syron, Johnell Fleming, Harold Magazine, Rick Hasz, Gary D. Walters, Jason P. Bridge, Mark Miklos, Susan Sullivan, Laura K. Barker, Heather M. Traino, Maghboeba Mosavel, Laura A. Siminoff, Dana R. Valley, Daniel C. Rohrer, Scott D. Jewell, Philip A. Branton, Leslie H. Sobin, Mary Barcus, Liqun Qi, Jeffrey McLean, Pushpa Hariharan, Ki Sung Um, Shenpei Wu, David Tabor, Charles Shive, Anna M. Smith, Stephen A. Buia, Anita H. Undale, Karna L. Robinson, Nancy Roche, Kimberly M. Valentino, Angela Britton, Robin Burges, Debra Bradbury, Kenneth W. Hambright, John Seleski, Greg E. Korzeniewski, Kenyon Erickson, Yvonne Marcus, Jorge Tejada, Mehran Taherian, Chunrong Lu, Margaret Basile, Deborah C. Mash, Simona Volpi, Jeffery P. Struewing, Gary F. Temple, Joy Boyer, Deborah Colantuoni, Roger Little, Susan Koester, Latarsha J. Carithers, Helen M. Moore, Ping Guan, Carolyn Compton, Sherilyn J. Sawyer, Joanne P. Demchok, Jimmie B. Vaught, Chana A. Rabiner, and Lockhart. 2015. "The Genotype-Tissue Expression (GTEx) Pilot Analysis: Multitissue Gene Regulation in Humans." Science.

Ashburner, M., C. A. Ball, J. A. Blake, D. Botstein, H. Butler, J. M. Cherry, A. P. Davis, K. Dolinski, S. S. Dwight, J. T. Eppig, M. A. Harris, D. P. Hill, L. Issel-Tarver, A. Kasarskis, S. Lewis, J. C. Matese, J. E. Richardson, M. Ringwald, G. M. Rubin, and G. Sherlock. 2000. "Gene Ontology: Tool for the Unification of Biology. The Gene Ontology Consortium.” Nature Genetics 25(1):25-29.

Balagopal, Vidya and Karen L. Beemon. 2017. "Rous Sarcoma Virus RNA Stability Element Inhibits Deadenylation of MRNAs with Long 3'UTRs." Viruses 9(8).

Bao, Jianqiang, Kristoffer Vitting-Seerup, Johannes Waage, Chong Tang, Ying Ge, Bo T. Porse, and Wei Yan. 2016. "UPF2-Dependent Nonsense-Mediated MRNA Decay Pathway Is Essential for 
Spermatogenesis by Selectively Eliminating Longer 3'UTR Transcripts.” PLoS Genetics 12(5).

Behm-Ansmant, Isabelle, David Gatfield, Jan Rehwinkel, Valérie Hilgers, and Elisa Izaurralde. 2007. “A Conserved Role for Cytoplasmic Poly(A)-Binding Protein 1 (PABPC1) in Nonsense-Mediated MRNA Decay." The EMBO Journal 26(6):1591-1601.

Bejerano, Gill, Michael Pheasant, Igor Makunin, Stuart Stephen, W. James Kent, John S. Mattick, and David Haussler. 2004. "Ultraconserved Elements in the Human Genome." Science (New York, N.Y.) 304(5675):1321-25.

Bhattacharya, A., K. Czaplinski, P. Trifillis, F. He, A. Jacobson, and S. W. Peltz. 2000. "Characterization of the Biochemical Properties of the Human Upfl Gene Product That Is Involved in NonsenseMediated MRNA Decay." RNA (New York, N.Y.) 6(9):1226-35.

Brooks, Angela N., Li Yang, Michael O. Duff, Kasper D. Hansen, Jung W. Park, Sandrine Dudoit, Steven E. Brenner, and Brenton R. Graveley. 2011. "Conservation of an RNA Regulatory Map between Drosophila and Mammals.” Genome Research 21(2):193-202.

Bruno, Ivone G., Rachid Karam, Lulu Huang, Anjana Bhardwaj, Chih H. Lou, Eleen Y. Shum, Hye-Won Song, Mark A. Corbett, Wesley D. Gifford, Jozef Gecz, Samuel L. Pfaff, and Miles F. Wilkinson. 2011. "Identification of a MicroRNA That Activates Gene Expression by Repressing NonsenseMediated RNA Decay." Molecular Cell 42(4):500-510.

Bühler, Marc, Silvia Steiner, Fabio Mohn, Alexandra Paillusson, and Oliver Mühlemann. 2006. "EJCIndependent Degradation of Nonsense Immunoglobulin-Mu MRNA Depends on 3' UTR Length." Nature Structural \& Molecular Biology 13(5):462-64.

Chakrabarti, Sutapa, Fabien Bonneau, Steffen Schüssler, Elfriede Eppinger, and Elena Conti. 2014. "Phospho-Dependent and Phospho-Independent Interactions of the Helicase UPF1 with the NMD Factors SMG5-SMG7 and SMG6." Nucleic Acids Research 42(14):9447-60.

Chakrabarti, Sutapa, Uma Jayachandran, Fabien Bonneau, Francesca Fiorini, Claire Basquin, Silvia Domcke, Hervé Le Hir, and Elena Conti. 2011. "Molecular Mechanisms for the RNA-Dependent ATPase Activity of Upf1 and Its Regulation by Upf2." Molecular Cell 41(6):693-703.

Chamieh, Hala, Lionel Ballut, Fabien Bonneau, and Hervé Le Hir. 2008. "NMD Factors UPF2 and UPF3 Bridge UPF1 to the Exon Junction Complex and Stimulate Its RNA Helicase Activity." Nature Structural \& Molecular Biology 15(1):85-93.

Colombo, Martino, Evangelos D. Karousis, Joël Bourquin, Rémy Bruggmann, and Oliver Mühlemann. 2017. "Transcriptome-Wide Identification of NMD-Targeted Human MRNAs Reveals Extensive Redundancy between SMG6- and SMG7-Mediated Degradation Pathways.” RNA.

Cuccurese, Monica, Giulia Russo, Annapina Russo, and Concetta Pietropaolo. 2005. "Alternative Splicing and Nonsense-Mediated MRNA Decay Regulate Mammalian Ribosomal Gene Expression." Nucleic Acids Research 33(18):5965-77.

Deniaud, Aurélien, Manikandan Karuppasamy, Thomas Bock, Simonas Masiulis, Karine Huard, Frédéric Garzoni, Kathrin Kerschgens, Matthias W. Hentze, Andreas E. Kulozik, Martin Beck, Gabriele NeuYilik, and Christiane Schaffitzel. 2015. "A Network of SMG-8, SMG-9 and SMG-1 C-Terminal Insertion Domain Regulates UPF1 Substrate Recruitment and Phosphorylation.” Nucleic Acids 
Research 43(15):7600-7611.

Dredge, B. Kate and Kirk B. Jensen. 2011. "NeuN/Rbfox3 Nuclear and Cytoplasmic Isoforms Differentially Regulate Alternative Splicing and Nonsense-Mediated Decay of Rbfox2." PloS One 6(6):e21585.

Dyle, Michael C., Divya Kolakada, Michael A. Cortazar, and Sujatha Jagannathan. 2019. "How to Get Away with Nonsense: Mechanisms and Consequences of Escape from Nonsense-mediated RNA Decay." Wiley Interdisciplinary Reviews: RNA.

Eberle, Andrea B., Lukas Stalder, Hansruedi Mathys, Rodolfo Zamudio Orozco, and Oliver Mühlemann. 2008. "Posttranscriptional Gene Regulation by Spatial Rearrangement of the 3' Untranslated Region.” PLoS Biology 6(4):e92.

Fanourgakis, Grigorios, Mathias Lesche, Müge Akpinar, Andreas Dahl, and Rolf Jessberger. 2016. “Chromatoid Body Protein TDRD6 Supports Long 3' UTR Triggered Nonsense Mediated MRNA Decay." PLoS Genetics 12(5):e1005857.

Fernandes, Rafael, Gonçalo Nogueira, Paulo J. da Costa, Francisco Pinto, and Luísa Romão. 2019. "Nonsense-Mediated MRNA Decay in Development, Stress and Cancer." Pp. 41-83 in Advances in experimental medicine and biology. Vol. 1157.

Frischmeyer-Guerrerio, Pamela A., Robert A. Montgomery, Daniel S. Warren, Sara K. Cooke, Johannes Lutz, Christopher J. Sonnenday, Anthony L. Guerrerio, and Harry C. Dietz. 2011. "Perturbation of Thymocyte Development in Nonsense-Mediated Decay (NMD)-Deficient Mice." Proceedings of the National Academy of Sciences of the United States of America 108(26):10638-43.

Fujita, Pauline A., Brooke Rhead, Ann S. Zweig, Angie S. Hinrichs, Donna Karolchik, Melissa S. Cline, Mary Goldman, Galt P. Barber, Hiram Clawson, Antonio Coelho, Mark Diekhans, Timothy R. Dreszer, Belinda M. Giardine, Rachel A. Harte, Jennifer Hillman-Jackson, Fan Hsu, Vanessa Kirkup, Robert M. Kuhn, Katrina Learned, Chin H. Li, Laurence R. Meyer, Andy Pohl, Brian J. Raney, Kate R. Rosenbloom, Kayla E. Smith, David Haussler, and W. James Kent. 2011. "The UCSC Genome Browser Database: Update 2011.” Nucleic Acids Research 39(Database issue):D876-82.

Ge, Zhiyun, Bao Lin Quek, Karen L. Beemon, and J. Robert Hogg. 2016. "Polypyrimidine Tract Binding Protein 1 Protects MRNAs from Recognition by the Nonsense-Mediated MRNA Decay Pathway." ELife 5.

Gehring, Niels H., Joachim B. Kunz, Gabriele Neu-Yilik, Stephen Breit, Marcelo H. Viegas, Matthias W. Hentze, and Andreas E. Kulozik. 2005. "Exon-Junction Complex Components Specify Distinct Routes of Nonsense-Mediated MRNA Decay with Differential Cofactor Requirements." Molecular Cell 20(1):65-75.

Gilat, Roi and Dorit Shweiki. 2007. “A Novel Function for Alternative Polyadenylation as a Rescue Pathway from NMD Surveillance." Biochemical and Biophysical Research Communications 353(2):487-92.

Hansen, Kasper Daniel, Liana F. Lareau, Marco Blanchette, Richard E. Green, Qi Meng, Jan Rehwinkel, Fabian L. Gallusser, Elisa Izaurralde, Donald C. Rio, Sandrine Dudoit, and Steven E. Brenner. 2009. "Genome-Wide Identification of Alternative Splice Forms down-Regulated by Nonsense-Mediated 
MRNA Decay in Drosophila.” PLoS Genetics 5(6):e1000525.

Harte, Rachel A., Catherine M. Farrell, Jane E. Loveland, Marie-Marthe Suner, Laurens Wilming, Bronwen Aken, Daniel Barrell, Adam Frankish, Craig Wallin, Steve Searle, Mark Diekhans, Jennifer Harrow, and Kim D. Pruitt. 2012. "Tracking and Coordinating an International Curation Effort for the CCDS Project." Database : The Journal of Biological Databases and Curation 2012:bas008.

Hatano, Masaya, Mariko Umemura, Natsumi Kimura, Takashi Yamazaki, Hitoshi Takeda, Haruo Nakano, Shigeru Takahashi, and Yuji Takahashi. 2013. "The 5'-Untranslated Region Regulates ATF5 MRNA Stability via Nonsense-Mediated MRNA Decay in Response to Environmental Stress.” The FEBS Journal 280(18):4693-4707.

He, F., S. W. Peltz, J. L. Donahue, M. Rosbash, and A. Jacobson. 1993. "Stabilization and Ribosome Association of Unspliced Pre-MRNAs in a Yeast Upf1 - Mutant." Proceedings of the National Academy of Sciences of the United States of America 90(15):7034-38.

He, Feng and Allan Jacobson. 2015. "Nonsense-Mediated MRNA Decay: Degradation of Defective Transcripts Is Only Part of the Story." Annual Review of Genetics 49:339-66.

Hoek, Tim A., Deepak Khuperkar, Rik G. H. Lindeboom, Stijn Sonneveld, Bram M. P. Verhagen, Sanne Boersma, Michiel Vermeulen, and Marvin E. Tanenbaum. 2019. "Single-Molecule Imaging Uncovers Rules Governing Nonsense-Mediated MRNA Decay.” Molecular Cell 75(2):324-339.e11.

Hogg, J. Robert and Stephen P. Goff. 2010. "Upf1 Senses 3'UTR Length to Potentiate MRNA Decay.” Cell 143(3):379-89.

Huang, Da Wei, Brad T. Sherman, Qina Tan, Jack R. Collins, W. Gregory Alvord, Jean Roayaei, Robert Stephens, Michael W. Baseler, H. Clifford Lane, and Richard A. Lempicki. 2007. "The DAVID Gene Functional Classification Tool: A Novel Biological Module-Centric Algorithm to Functionally Analyze Large Gene Lists." Genome Biology.

Huang, Lulu, Chih-Hong Lou, Waikin Chan, Eleen Y. Shum, Ada Shao, Erica Stone, Rachid Karam, HyeWon Song, and Miles F. Wilkinson. 2011. "RNA Homeostasis Governed by Cell Type-Specific and Branched Feedback Loops Acting on NMD." Molecular Cell 43(6):950-61.

Huntzinger, Eric, Isao Kashima, Maria Fauser, Jérôme Saulière, and Elisa Izaurralde. 2008. "SMG6 Is the Catalytic Endonuclease That Cleaves MRNAs Containing Nonsense Codons in Metazoan." RNA (New York, N.Y.) 14(12):2609-17.

Hurt, Jessica A., Alex D. Robertson, and Christopher B. Burge. 2013. "Global Analyses of UPF1 Binding and Function Reveal Expanded Scope of Nonsense-Mediated MRNA Decay." Genome Research 23(10):1636-50.

Hwang, Jungwook, Hanae Sato, Yalan Tang, Daiki Matsuda, and Lynne E. Maquat. 2010. “UPF1 Association with the Cap-Binding Protein, CBP80, Promotes Nonsense-Mediated MRNA Decay at Two Distinct Steps.” Molecular Cell 39(3):396-409.

Hyvönen, Mervi T., Anne Uimari, Tuomo A. Keinänen, Sami Heikkinen, Riikka Pellinen, Tiina Wahlfors, Arja Korhonen, Ale Närvänen, Jarmo Wahlfors, Leena Alhonen, and Juhani Jänne. 2006. "Polyamine-Regulated Unproductive Splicing and Translation of Spermidine/Spermine N1Acetyltransferase." RNA (New York, N.Y.) 12(8):1569-82. 
Hyvönen, Mervi T., Anne Uimari, Jouko Vepsäläinen, Alex R. Khomutov, Tuomo A. Keinänen, and Leena Alhonen. 2012. "Tissue-Specific Alternative Splicing of Spermidine/Spermine N

(1)-Acetyltransferase." Amino Acids 42(2-3):485-93.

Imamachi, Naoto, Kazi Abdus Salam, Yutaka Suzuki, and Nobuyoshi Akimitsu. 2016. "A GC-Rich Sequence Feature in the 3' UTR Directs UPF1-Dependent MRNA Decay in Mammalian Cells." Genome Research.

Isken, Olaf and Lynne E. Maquat. 2007. "Quality Control of Eukaryotic MRNA: Safeguarding Cells from Abnormal MRNA Function.” Genes \& Development 21(15):1833-56.

Ivanov, Pavel V, Niels H. Gehring, Joachim B. Kunz, Matthias W. Hentze, and Andreas E. Kulozik. 2008. "Interactions between UPF1, ERFs, PABP and the Exon Junction Complex Suggest an Integrated Model for Mammalian NMD Pathways." The EMBO Journal 27(5):736-47.

Jangi, Mohini, Paul L. Boutz, Prakriti Paul, and Phillip A. Sharp. 2014. "Rbfox2 Controls Autoregulation in RNA-Binding Protein Networks." Genes \& Development 28(6):637-51.

Jumaa, H. and P. J. Nielsen. 1997. "The Splicing Factor SRp20 Modifies Splicing of Its Own MRNA and ASF/SF2 Antagonizes This Regulation.” The EMBO Journal 16(16):5077-85.

Karam, Rachid and Miles Wilkinson. 2012. “A Conserved MicroRNA/NMD Regulatory Circuit Controls Gene Expression.” RNA Biology 9(1).

Kashima, Isao, Akio Yamashita, Natsuko Izumi, Naoyuki Kataoka, Ryo Morishita, Shinichi Hoshino, Mutsuhito Ohno, Gideon Dreyfuss, and Shigeo Ohno. 2006. "Binding of a Novel SMG-1-Upf1ERF1-ERF3 Complex (SURF) to the Exon Junction Complex Triggers Upf1 Phosphorylation and Nonsense-Mediated MRNA Decay." Genes \& Development 20(3):355-67.

Kaygun, Handan and William F. Marzluff. 2005. "Regulated Degradation of Replication-Dependent Histone MRNAs Requires Both ATR and Upf1.” Nature Structural \& Molecular Biology 12(9):794800.

Kerényi, Zoltán, Zsuzsanna Mérai, László Hiripi, Anna Benkovics, Péter Gyula, Christophe Lacomme, Endre Barta, Ferenc Nagy, and Dániel Silhavy. 2008. "Inter-Kingdom Conservation of Mechanism of Nonsense-Mediated MRNA Decay.” The EMBO Journal 27(11):1585-95.

Kervestin, Stephanie and Allan Jacobson. 2012. "NMD: A Multifaceted Response to Premature Translational Termination.” Nature Reviews. Molecular Cell Biology 13(11):700-712.

Kervestin, Stephanie, Chunfang Li, Richard Buckingham, and Allan Jacobson. 2012. "Testing the FauxUTR Model for NMD: Analysis of Upf1p and Pab1p Competition for Binding to ERF3/Sup35p." Biochimie.

Kim, Yoon Ki, Luc Furic, Luc DesGroseillers, and Lynne E. Maquat. 2005. "Mammalian Staufen1 Recruits Upf1 to Specific MRNA 3'UTRs so as to Elicit MRNA Decay.” Cell 120(2):195-208.

Kishor, Aparna, Sarah E. Fritz, and J. Robert Hogg. 2019. "Nonsense-mediated MRNA Decay: The Challenge of Telling Right from Wrong in a Complex Transcriptome." Wiley Interdisciplinary Reviews: RNA e1548. 
Kishor, Aparna, Zhiyun Ge, and J. Robert Hogg. 2019. "HnRNP L-Dependent Protection of Normal MRNAs from NMD Subverts Quality Control in B Cell Lymphoma.” The EMBO Journal 38(3).

Kozak, M. 1991. "Structural Features in Eukaryotic MRNAs That Modulate the Initiation of Translation." The Journal of Biological Chemistry 266(30):19867-70.

Kurosaki, Tatsuaki and Lynne E. Maquat. 2013. "Rules That Govern UPF1 Binding to MRNA 3' UTRs." Proc Natl Acad Sci USA 110(9):3357-62.

Kurosaki, Tatsuaki, Maximilian W. Popp, and Lynne E. Maquat. 2019. "Quality and Quantity Control of Gene Expression by Nonsense-Mediated MRNA Decay." Nature Reviews Molecular Cell Biology 20(7):406-20.

Langmead, Ben, Cole Trapnell, Mihai Pop, and Steven L. Salzberg. 2009. "Ultrafast and MemoryEfficient Alignment of Short DNA Sequences to the Human Genome.” Genome Biology 10(3):R25.

Lareau, Liana F. and Steven E. Brenner. 2015. "Regulation of Splicing Factors by Alternative Splicing and NMD Is Conserved between Kingdoms Yet Evolutionarily Flexible." Molecular Biology and Evolution 32(4):1072-79.

Lareau, Liana F., Angela N. Brooks, David A. W. Soergel, Qi Meng, and Steven E. Brenner. 2007. "The Coupling of Alternative Splicing and Nonsense-Mediated MRNA Decay." Advances in Experimental Medicine and Biology 623:190-211.

Lareau, Liana F., Maki Inada, Richard E. Green, Jordan C. Wengrod, and Steven E. Brenner. 2007. "Unproductive Splicing of SR Genes Associated with Highly Conserved and Ultraconserved DNA Elements." Nature 446(7138):926-29.

Lasalde, Clarivel, Andrea V Rivera, Alfredo J. León, José A. González-Feliciano, Luis A. Estrella, Eva N. Rodríguez-Cruz, María E. Correa, Iván J. Cajigas, Dina P. Bracho, Irving E. Vega, Miles F. Wilkinson, and Carlos I. González. 2014. "Identification and Functional Analysis of Novel Phosphorylation Sites in the RNA Surveillance Protein Upf1." Nucleic Acids Research 42(3):191629.

Lewis, Benjamin P., Richard E. Green, and Steven E. Brenner. 2003. "Evidence for the Widespread Coupling of Alternative Splicing and Nonsense-Mediated MRNA Decay in Humans." Proceedings of the National Academy of Sciences of the United States of America 100(1):189-92.

Lindeboom, Rik G. H., Fran Supek, and Ben Lehner. 2016. "The Rules and Impact of Nonsense-Mediated MRNA Decay in Human Cancers." Nature Genetics 48(10):1112-18.

Lloyd, James P. B. 2018. "The Evolution and Diversity of the Nonsense-Mediated MRNA Decay Pathway." F1000Research 7:1299.

Lloyd, James P. B., Courtney E. French, and Steven E. Brenner. 2020. "Polysome Fractionation Analysis Reveals Features Important for Human Nonsense-Mediated MRNA Decay." BioRxiv 2020.03.08.981811.

Lloyd, James P. B., Daniel Lang, Andreas D. Zimmer, Barry Causier, Ralf Reski, and Brendan Davies. 2018. "The Loss of SMG1 Causes Defects in Quality Control Pathways in Physcomitrella Patens." Nucleic Acids Research 46(11):5822-36. 
Lou, Chih Hong, Jennifer Dumdie, Alexandra Goetz, Eleen Y. Shum, David Brafman, Xiaoyan Liao, Sergio Mora-Castilla, Madhuvanthi Ramaiah, Heidi Cook-Andersen, Louise Laurent, and Miles F. Wilkinson. 2016. "Nonsense-Mediated RNA Decay Influences Human Embryonic Stem Cell Fate." Stem Cell Reports 6(6):844-57.

Lykke-Andersen, J. and E. J. Bennett. 2014. "Protecting the Proteome: Eukaryotic Cotranslational Quality Control Pathways." The Journal of Cell Biology.

Lykke-Andersen, Jens, Mei-Di Shu, and Joan A. Steitz. 2000. "Human Upf Proteins Target an MRNA for Nonsense-Mediated Decay When Bound Downstream of a Termination Codon." Cell 103(7):112131.

Lykke-Andersen, Søren, Yun Chen, Britt R. Ardal, Berit Lilje, Johannes Waage, Albin Sandelin, and Torben Heick Jensen. 2014. "Human Nonsense-Mediated RNA Decay Initiates Widely by Endonucleolysis and Targets SnoRNA Host Genes." Genes \& Development 28(22):2498-2517.

Lykke-Andersen, Søren and Torben Heick Jensen. 2015. "Nonsense-Mediated MRNA Decay: An Intricate Machinery That Shapes Transcriptomes." Nature Reviews. Molecular Cell Biology 16(11):665-77.

McGlincy, Nicholas J., Lit-Yeen Tan, Nicodeme Paul, Mihaela Zavolan, Kathryn S. Lilley, and Christopher W. J. Smith. 2010. "Expression Proteomics of UPF1 Knockdown in HeLa Cells Reveals Autoregulation of HnRNP A2/B1 Mediated by Alternative Splicing Resulting in Nonsense-Mediated MRNA Decay." BMC Genomics 11:565.

Melero, Roberto, Akiko Uchiyama, Raquel Castaño, Naoyuki Kataoka, Hitomi Kurosawa, Shigeo Ohno, Akio Yamashita, and Oscar Llorca. 2014. "Structures of SMG1-UPFs Complexes: SMG1 Contributes to Regulate UPF2-Dependent Activation of UPF1 in NMD." Structure (London, England: 1993) 22(8):1105-19.

Mendell, Joshua T., Neda A. Sharifi, Jennifer L. Meyers, Francisco Martinez-Murillo, and Harry C. Dietz. 2004. "Nonsense Surveillance Regulates Expression of Diverse Classes of Mammalian Transcripts and Mutes Genomic Noise." Nature Genetics 36(10):1073-78.

Metze, Stefanie, Veronika A. Herzog, Marc-David Ruepp, and Oliver Mühlemann. 2013. "Comparison of EJC-Enhanced and EJC-Independent NMD in Human Cells Reveals Two Partially Redundant Degradation Pathways." RNA (New York, N.Y.) 19(10):1432-48.

Moriarty, P. M., C. C. Reddy, and L. E. Maquat. 1998. "Selenium Deficiency Reduces the Abundance of MRNA for Se-Dependent Glutathione Peroxidase 1 by a UGA-Dependent Mechanism Likely to Be Nonsense Codon-Mediated Decay of Cytoplasmic MRNA.” Molecular and Cellular Biology 18(5):2932-39.

Mudge, Jonathan M., Adam Frankish, Julio Fernandez-Banet, Tyler Alioto, Thomas Derrien, Cédric Howald, Alexandre Reymond, Roderic Guigó, Tim Hubbard, and Jennifer Harrow. 2011. "The Origins, Evolution, and Functional Potential of Alternative Splicing in Vertebrates." Molecular Biology and Evolution 28(10):2949-59.

Nagy, E. and L. E. Maquat. 1998. "A Rule for Termination-Codon Position within Intron-Containing Genes: When Nonsense Affects RNA Abundance.” Trends in Biochemical Sciences 23(6):198-99.

Nelson, Jonathan O., Kristin A. Moore, Alex Chapin, Julie Hollien, and Mark M. Metzstein. 2016. 
“Degradation of Gadd45 MRNA by Nonsense-Mediated Decay Is Essential for Viability.” ELife 5.

Neu-Yilik, Gabriele, Beate Amthor, Niels H. Gehring, Sharif Bahri, Helena Paidassi, Matthias W. Hentze, and Andreas E. Kulozik. 2011. "Mechanism of Escape from Nonsense-Mediated MRNA Decay of Human Beta-Globin Transcripts with Nonsense Mutations in the First Exon." RNA (New York, N.Y.) 17(5):843-54.

Ni, Julie Z., Leslie Grate, John Paul Donohue, Christine Preston, Naomi Nobida, Georgeann O’Brien, Lily Shiue, Tyson A. Clark, John E. Blume, and Manuel Ares. 2007. "Ultraconserved Elements Are Associated with Homeostatic Control of Splicing Regulators by Alternative Splicing and NonsenseMediated Decay." Genes \& Development 21(6):708-18.

Nicholson, Pamela, Christoph Josi, Hitomi Kurosawa, Akio Yamashita, and Oliver Mühlemann. 2014. “A Novel Phosphorylation-Independent Interaction between SMG6 and UPF1 Is Essential for Human NMD." Nucleic Acids Research 42(14):9217-35.

Nyikó, Tünde, Andor Auber, Levente Szabadkai, Anna Benkovics, Mariann Auth, Zsuzsanna Mérai, Zoltán Kerényi, Andrea Dinnyés, Ferenc Nagy, and Dániel Silhavy. 2017. "Expression of the ERF1 Translation Termination Factor Is Controlled by an Autoregulatory Circuit Involving Readthrough and Nonsense-Mediated Decay in Plants.” Nucleic Acids Research 45(7):4174-88.

Nyikó, Tünde, Boglárka Sonkoly, Zsuzsanna Mérai, Anna Hangyáné Benkovics, and Dániel Silhavy. 2009. "Plant Upstream ORFs Can Trigger Nonsense-Mediated MRNA Decay in a Size-Dependent Manner.” Plant Molecular Biology 71(4-5):367-78.

Okada-Katsuhata, Yukiko, Akio Yamashita, Kei Kutsuzawa, Natsuko Izumi, Fumiki Hirahara, and Shigeo Ohno. 2012. "N- and C-Terminal Upf1 Phosphorylations Create Binding Platforms for SMG-6 and SMG-5:SMG-7 during NMD.” Nucleic Acids Research 40(3):1251-66.

Paillusson, Alexandra, Nadine Hirschi, Claudio Vallan, Claus M. Azzalin, and Oliver Mühlemann. 2005. "A GFP-Based Reporter System to Monitor Nonsense-Mediated MRNA Decay." Nucleic Acids Research 33(6):e54.

Pan, Qun, Arneet L. Saltzman, Yoon Ki Kim, Christine Misquitta, Ofer Shai, Lynne E. Maquat, Brendan J. Frey, and Benjamin J. Blencowe. 2006. "Quantitative Microarray Profiling Provides Evidence against Widespread Coupling of Alternative Splicing with Nonsense-Mediated MRNA Decay to Control Gene Expression." Genes \& Development 20(2):153-58.

Peixeiro, Isabel, Angela Inácio, Cristina Barbosa, Ana Luísa Silva, Stephen A. Liebhaber, and Luísa Romão. 2012. "Interaction of PABPC1 with the Translation Initiation Complex Is Critical to the NMD Resistance of AUG-Proximal Nonsense Mutations." Nucleic Acids Research 40(3):1160-73.

Pervouchine, Dmitri, Yaroslav Popov, Andy Berry, Beatrice Borsari, Adam Frankish, and Roderic Guigó. 2019. "Integrative Transcriptomic Analysis Suggests New Autoregulatory Splicing Events Coupled with Nonsense-Mediated MRNA Decay.” Nucleic Acids Research 47(10):5293-5306.

Pimentel, Harold, Marilyn Parra, Sherry Gee, Dana Ghanem, Xiuli An, Jie Li, Narla Mohandas, Lior Pachter, and John G. Conboy. 2014. "A Dynamic Alternative Splicing Program Regulates Gene Expression during Terminal Erythropoiesis." Nucleic Acids Research 42(6):4031-42.

Plocik, Alex M. and Christine Guthrie. 2012. "Diverse Forms of RPS9 Splicing Are Part of an Evolving 
Autoregulatory Circuit.” PLoS Genetics 8(3):e1002620.

Pruitt, Kim D., Tatiana Tatusova, William Klimke, and Donna R. Maglott. 2009. "NCBI Reference Sequences: Current Status, Policy and New Initiatives.” Nucleic Acids Research 37(Database issue):D32-6.

Pulak, R. and P. Anderson. 1993. "MRNA Surveillance by the Caenorhabditis Elegans Smg Genes." Genes \& Development.

Rehwinkel, Jan, Ivica Letunic, Jeroen Raes, Peer Bork, and Elisa Izaurralde. 2005. "Nonsense-Mediated MRNA Decay Factors Act in Concert to Regulate Common MRNA Targets." RNA (New York, N.Y.) 11(10):1530-44.

Roberts, Adam, Harold Pimentel, Cole Trapnell, and Lior Pachter. 2011. "Identification of Novel Transcripts in Annotated Genomes Using RNA-Seq." Bioinformatics (Oxford, England) 27(17):2325-29.

Rossbach, Oliver, Lee-Hsueh Hung, Silke Schreiner, Inna Grishina, Monika Heiner, Jingyi Hui, and Albrecht Bindereif. 2009. "Auto- and Cross-Regulation of the HnRNP L Proteins by Alternative Splicing." Molecular and Cellular Biology 29(6):1442-51.

Russo, Annapina, Morena Catillo, Davide Esposito, Paola Briata, Concetta Pietropaolo, and Giulia Russo. 2011. "Autoregulatory Circuit of Human RpL3 Expression Requires HnRNP H1, NPM and KHSRP.” Nucleic Acids Research 39(17):7576-85.

Saltzman, Arneet L., Yoon Ki Kim, Qun Pan, Matthew M. Fagnani, Lynne E. Maquat, and Benjamin J. Blencowe. 2008. "Regulation of Multiple Core Spliceosomal Proteins by Alternative SplicingCoupled Nonsense-Mediated MRNA Decay." Molecular and Cellular Biology 28(13):4320-30.

Saltzman, Arneet L., Qun Pan, and Benjamin J. Blencowe. 2011. "Regulation of Alternative Splicing by the Core Spliceosomal Machinery." Genes \& Development 25(4):373-84.

Saulière, Jérôme, Nazmul Haque, Scot Harms, Isabelle Barbosa, Marco Blanchette, and Hervé Le Hir. 2010. "The Exon Junction Complex Differentially Marks Spliced Junctions.” Nature Structural \& Molecular Biology 17(10):1269-71.

Sayani, Shakir, Michael Janis, Chrissie Young Lee, Isabelle Toesca, and Guillaume F. Chanfreau. 2008. "Widespread Impact of Nonsense-Mediated MRNA Decay on the Yeast Intronome." Molecular Cell 31(3):360-70.

Schweingruber, Christoph, Simone C. Rufener, David Zünd, Akio Yamashita, and Oliver Mühlemann. 2013. "Nonsense-Mediated MRNA Decay - Mechanisms of Substrate MRNA Recognition and Degradation in Mammalian Cells." Biochimica et Biophysica Acta 1829(6-7):612-23.

Seyedali, Ali and Marla J. Berry. 2014. "Nonsense-Mediated Decay Factors Are Involved in the Regulation of Selenoprotein MRNA Levels during Selenium Deficiency." RNA (New York, N.Y.) 20(8):1248-56.

Singh, Guramrit, Indrani Rebbapragada, and Jens Lykke-Andersen. 2008. "A Competition between Stimulators and Antagonists of Upf Complex Recruitment Governs Human Nonsense-Mediated MRNA Decay." PLoS Biology 6(4):e111. 
Spellman, Rachel, Miriam Llorian, and Christopher W. J. Smith. 2007. "Crossregulation and Functional Redundancy between the Splicing Regulator PTB and Its Paralogs NPTB and ROD1." Molecular Cell 27(3):420-34.

Sun, Shuying, Zuo Zhang, Rahul Sinha, Rotem Karni, and Adrian R. Krainer. 2010. "SF2/ASF Autoregulation Involves Multiple Layers of Post-Transcriptional and Translational Control." Nature Structural \& Molecular Biology 17(3):306-12.

Sureau, A., R. Gattoni, Y. Dooghe, J. Stévenin, and J. Soret. 2001. "SC35 Autoregulates Its Expression by Promoting Splicing Events That Destabilize Its MRNAs.” The EMBO Journal 20(7):1785-96.

Tani, Hidenori, Naoto Imamachi, Kazi Abdus Salam, Rena Mizutani, Kenichi Ijiri, Takuma Irie, Tetsushi Yada, Yutaka Suzuki, and Nobuyoshi Akimitsu. 2012. "Identification of Hundreds of Novel UPF1 Target Transcripts by Direct Determination of Whole Transcriptome Stability." RNA Biology 9(11):1370-79.

Taylor, Martin S., John LaCava, Paolo Mita, Kelly R. Molloy, Cheng Ran Lisa Huang, Donghui Li, Emily M. Adney, Hua Jiang, Kathleen H. Burns, Brian T. Chait, Michael P. Rout, Jef D. Boeke, and Lixin Dai. 2013. "Affinity Proteomics Reveals Human Host Factors Implicated in Discrete Stages of LINE-1 Retrotransposition.” Cell 155(5):1034-48.

Toma, Kalodiah G., Indrani Rebbapragada, Sébastien Durand, and Jens Lykke-Andersen. 2015. "Identification of Elements in Human Long 3' UTRs That Inhibit Nonsense-Mediated Decay." RNA (New York, N.Y.) 21(5):887-97.

Trapnell, Cole, Lior Pachter, and Steven L. Salzberg. 2009. “TopHat: Discovering Splice Junctions with RNA-Seq." Bioinformatics (Oxford, England) 25(9):1105-11.

Trapnell, Cole, Brian A. Williams, Geo Pertea, Ali Mortazavi, Gordon Kwan, Marijke J. van Baren, Steven L. Salzberg, Barbara J. Wold, and Lior Pachter. 2010. "Transcript Assembly and Quantification by RNA-Seq Reveals Unannotated Transcripts and Isoform Switching during Cell Differentiation." Nature Biotechnology 28(5):511-15.

Vigneault, Francois, A. Michael Sismour, and George M. Church. 2008. "Efficient MicroRNA Capture and Bar-Coding via Enzymatic Oligonucleotide Adenylation." Nature Methods.

Weischenfeldt, Joachim, Inge Damgaard, David Bryder, Kim Theilgaard-Mönch, Lina A. Thoren, Finn Cilius Nielsen, Sten Eirik W. Jacobsen, Claus Nerlov, and Bo Torben Porse. 2008. "NMD Is Essential for Hematopoietic Stem and Progenitor Cells and for Eliminating By-Products of Programmed DNA Rearrangements." Genes \& Development 22(10):1381-96.

Weischenfeldt, Joachim, Johannes E. Waage, Geng Tian, Jing Zhao, Inge Damgaard, Janus S. Jakobsen, Karsten Kristiansen, Anders Krogh, Jun Wang, and Bo T. Porse. 2012. "Mammalian Tissues Defective in Nonsense-Mediated MRNA Decay Display Highly Aberrant Splicing Patterns." Genome Biology 13(5):R35.

Wengrod, Jordan, Leenus Martin, Ding Wang, Pamela Frischmeyer-Guerrerio, Harry C. Dietz, and Lawrence B. Gardner. 2013. "Inhibition of Nonsense-Mediated RNA Decay Activates Autophagy." Molecular and Cellular Biology 33(11):2128-35.

Wittmann, Jürgen, Elly M. Hol, and Hans-Martin Jäck. 2006. "HUPF2 Silencing Identifies Physiologic 
Substrates of Mammalian Nonsense-Mediated MRNA Decay." Molecular and Cellular Biology 26(4):1272-87.

Wong, Justin J. L., William Ritchie, Olivia A. Ebner, Matthias Selbach, Jason W. H. Wong, Yizhou Huang, Dadi Gao, Natalia Pinello, Maria Gonzalez, Kinsha Baidya, Annora Thoeng, Teh-Liane Khoo, Charles G. Bailey, Jeff Holst, and John E. J. Rasko. 2013. "Orchestrated Intron Retention Regulates Normal Granulocyte Differentiation.” Cell 154(3):583-95.

Wright, Caroline F., Tomas W. Fitzgerald, Wendy D. Jones, Stephen Clayton, Jeremy F. McRae, Margriet Van Kogelenberg, Daniel A. King, Kirsty Ambridge, Daniel M. Barrett, Tanya Bayzetinova, A. Paul Bevan, Eugene Bragin, Eleni A. Chatzimichali, Susan Gribble, Philip Jones, Netravathi Krishnappa, Laura E. Mason, Ray Miller, Katherine I. Morley, Vijaya Parthiban, Elena Prigmore, Diana Rajan, Alejandro Sifrim, G. Jawahar Swaminathan, Adrian R. Tivey, Anna Middleton, Michael Parker, Nigel P. Carter, Jeffrey C. Barrett, Matthew E. Hurles, David R. Fitzpatrick, and Helen V Firth. 2015. "Genetic Diagnosis of Developmental Disorders in the DDD Study: A Scalable Analysis of Genome-Wide Research Data." The Lancet 385(9975):1305-14.

Yamashita, Akio, Natsuko Izumi, Isao Kashima, Tetsuo Ohnishi, Bonnie Saari, Yukiko Katsuhata, Reiko Muramatsu, Tomoko Morita, Akihiro Iwamatsu, Takahisa Hachiya, Rie Kurata, Hisashi Hirano, Philip Anderson, and Shigeo Ohno. 2009. "SMG-8 and SMG-9, Two Novel Subunits of the SMG-1 Complex, Regulate Remodeling of the MRNA Surveillance Complex during Nonsense-Mediated MRNA Decay." Genes \& Development 23(9):1091-1105.

Yan, Qinghong, Sebastien M. Weyn-Vanhentenryck, Jie Wu, Steven A. Sloan, Ye Zhang, Kenian Chen, Jia Qian Wu, Ben A. Barres, and Chaolin Zhang. 2015. "Systematic Discovery of Regulated and Conserved Alternative Exons in the Mammalian Brain Reveals NMD Modulating Chromatin Regulators." Proc Natl Acad Sci USA 112(11):3445-50.

Yepiskoposyan, Hasmik, Florian Aeschimann, Daniel Nilsson, Michal Okoniewski, and Oliver Mühlemann. 2011. "Autoregulation of the Nonsense-Mediated MRNA Decay Pathway in Human Cells.” RNA (New York, N.Y.) 17(12):2108-18.

Zhang, J. and L. E. Maquat. 1997. "Evidence That Translation Reinitiation Abrogates Nonsense-Mediated MRNA Decay in Mammalian Cells." The EMBO Journal 16(4):826-33.

Zheng, Sika, Erin E. Gray, Geetanjali Chawla, Bo Torben Porse, Thomas J. O'Dell, and Douglas L. Black. 2012. "PSD-95 Is Post-Transcriptionally Repressed during Early Neural Development by PTBP1 and PTBP2." Nature Neuroscience.

Zünd, David, Andreas R. Gruber, Mihaela Zavolan, and Oliver Mühlemann. 2013. "TranslationDependent Displacement of UPF1 from Coding Sequences Causes Its Enrichment in 3' UTRs." Nature Structural \& Molecular Biology 20(8):936-43. 